\title{
Determinants of Alanine Dipeptide Conformational Equilibria on Graphene and Hydroxylated Derivatives
}

\author{
Horacio Poblete, ${ }^{\dagger}$ Ingrid Miranda-Carvajal, ${ }^{\ddagger}$ and Jeffrey Comer ${ }^{*} \dagger$ (c) \\ ${ }^{\dagger}$ Institute of Computational Comparative Medicine, Nanotechnology Innovation Center of Kansas State, Department of Anatomy \\ and Physiology, Kansas State University, Manhattan, Kansas 66506-5802, United States \\ ‡Universidad Nacional de Colombia, sede Bogotá, Facultad de Ciencias, Departamento de Química, Carrera 30 No. 45-03, Bogotá, \\ 111321, Colombia
}

Supporting Information

ABSTRACT: Understanding the interaction of carbon nanomaterials with proteins is essential for determining the potential effects of these materials on health and in the design of biotechnology based on them. Here we leverage explicit-solvent molecular simulation and multidimensional freeenergy calculations to investigate how adsorption to carbon nanomaterial surfaces affects the conformational equilibrium of alanine dipeptide, a widely used model of protein backbone structure. We find that the two most favorable structures of alanine dipeptide on graphene (or large carbon nanotubes) correspond to the two amide linkages lying in the same plane, flat against the surface, rather than the nonplanar $\alpha$-helix-like and $\beta$ -

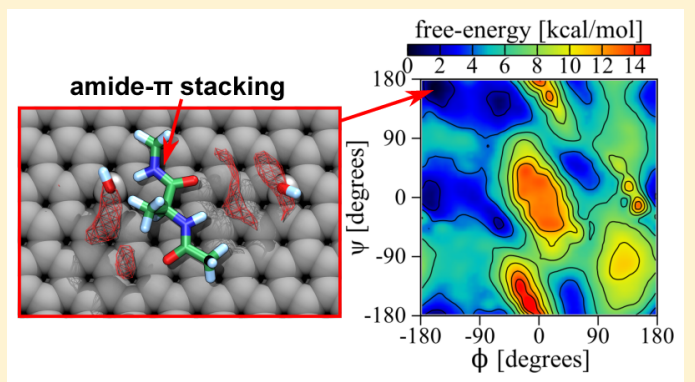
sheet-like conformations that predominate in aqueous solution. On graphenic surfaces, the latter conformations are metastable and most often correspond to amide $-\pi$ stacking of the $\mathrm{N}$-terminal amide. The calculations highlight the key role of amide $-\pi$ interactions in determining the conformational equilibrium. Lesser but significant contributions from hydrogen bonding to the high density interfacial water layer or to the hydroxy groups of hydroxylated graphene also define the most favorable conformations. This work should yield insight on the influence of carbon nanotubes, graphene, and their functionalized derivatives on protein structure.

\section{INTRODUCTION}

In biological environments, proteins bind to the surface of synthetic nanomaterials, forming a layer known as the protein corona that often determines the physiological distribution and biological activity of the materials ${ }^{1-8}$ and influences their toxicity $^{9-11}$ and immunogenicity. ${ }^{12-16}$ The composition of the corona depends on the nanomaterial's geometry, ${ }^{17}$ chemistry, surface modification, ${ }^{1}$ exposure time, ${ }^{10,18-21}$ and biological environment. $^{17,22,23}$ Thus, an understanding of engineered nanomaterial-protein interaction is essential for the design of nanomedicine and predicting the effects of nanomaterial exposure on humans and other organisms. Although the present work focuses on peptides and proteins, it should be noted that biomolecules other than proteins, such as lipids ${ }^{23,24}$ and sugars, ${ }^{25}$ can also be nanomaterial corona components, although their influence on biological activity has not been well studied thus far.

Owing to the large surface-area-to-volume ratio of nanostructures, which enhances the influence of surface interactions, most work on protein coronas has focused on nanomaterials. However, it should be pointed out that the biological importance of the protein corona does not apply only to nanostructures (objects with dimensions $<100 \mathrm{~nm}$ ) but also can have similar effects on the response of organisms to macroscopic objects, such as implants. ${ }^{26-28}$ Furthermore, studies of protein adsorption to nanoparticles can be directly compared to studies employing macroscopic surfaces of the same material in cases where the local environment of protein is similar, i.e., when the dimensions of the nanoparticles are considerably larger than the protein and the surface morphology of the nanoparticles and macroscopic surface are similar on the length scale of the protein. The present work focuses on multilayer graphenic surfaces, which serve equally as models of graphene sheets, graphene nanoplatelets larger than the adsorbates considered, and well dispersed carbon nanotubes of diameters greater than a few nanometers. ${ }^{29,30}$

Much remains unknown about the structure of the protein corona, the conformational equilibria of the proteins that make it up, and how these things depend on the physicochemical properties of the surface. In particular, adsorption to synthetic surfaces can lead to partial unfolding of proteins, ${ }^{31,32}$ affecting their function ${ }^{33-36}$ and potentially leading to inflammation. ${ }^{26,37}$ Therefore, experimental and computational methodologies have been applied to probe protein structural changes upon adsorption to synthetic materials, including those that form the basis of common engineered nanomaterials. For example,

Special Issue: Klaus Schulten Memorial Issue

Received: February 4, 2017

Revised: March 11, 2017

Published: March 14, 2017 
Burkett and $\operatorname{Read}^{38}$ used circular dichroism spectroscopy and nuclear magnetic resonance to measure the loss of $\alpha$-helical structure upon adsorption of peptides adsorbed to a silica surface, while Asuri et al. ${ }^{39}$ used similar techniques to study structural changes in protein enzymes covalently attached to carbon nanotubes. Fourier transform infrared spectroscopy (FTIR) has been used to reveal conformational changes in protein antigens adsorbed to aluminum-based vaccine adjuvants. ${ }^{40,41}$

The stability of protein structure is determined by physicochemistry of the surface of engineered materials. For instance, gold nanoparticles functionalized by negatively charged sulfate containing moieties caused cytochrome $c$ to lose its $\alpha$-helical structure, while similar nanoparticles functionalized by PEG did not lead to significant conformational changes. ${ }^{32}$ Furthermore, for a given surface, different proteins may also behave differently. For instance, infrared spectroscopy and atomic force microscopy, corroborated by activity assays, revealed that $\alpha$-chymotrypsin completely denatures on the surface of carbon nanotubes, abolishing its enzymatic activity, while the structure of soybean peroxidase is only moderately perturbed on this surface and $30 \%$ of its native activity is retained. ${ }^{33}$ Changes to enzyme function upon adsorption have also been studied by DeLong and co-workers, ${ }^{35}$ showing that activities of luciferase and $\beta$-galactosidase are decreased by adsorption to $\mathrm{ZnO}, \mathrm{Co}_{3} \mathrm{O}_{4}$, and $\mathrm{MgO}$ nanoparticles but increased in the presence of $\mathrm{NiO}$ nanoparticles.

Because of the high spatial and temporal resolution of atomistic molecular dynamics simulation, it has increasingly been leveraged for exploring interactions between biological molecules and synthetic surfaces, beginning with pioneering simulations by Klaus Schulten and co-workers on the interaction between gold and gold-binding peptides. ${ }^{42}$ Peptides found experimentally to specifically bind to particular materials, ${ }^{43,44}$ including gold, silver, titanium, and graphene, have been the subject of many of these simulations, ${ }^{42,45-49}$ with the goal of understanding the mechanisms of their specificity. Peptides such as these, as well as some proteins, are used to modify growth of nanomaterials during their synthesis to control the morphology of the resulting nanostructure. ${ }^{50-53}$ Poblete et al. $^{54}$ calculated the adsorption free energy on silver surfaces of 5-mer peptides used to facilitate the fabrication of silver nanoparticles and found a ranking of affinities for different sequences in agreement with experiment.

The study of complete proteins is made difficult by the computational expense of the large systems required to accommodate these proteins and the long time scales required for observing large conformational changes. Recently, Derr et $\mathrm{al}^{55}$ simulated the adsorption of the digestive enzyme chymotrypsin to $\mathrm{SiO}_{2}$ and $\mathrm{TiO}_{2}$ nanoparticles using implicit solvent, showing similar adsorption free energies between the two materials but different protein surface densities at saturation. Brancolini et al. ${ }^{56}$ used a mix of docking and explicit-solvent simulation to determine the preferred orientation of ubiquitin on a gold surface and the contribution of specific amino acids. In the presence of a hydrodynamic flow, Carr et al. ${ }^{57}$ determined favorable interactions between a toxin protein and amorphous silica. However, the time required for such large proteins to fold and unfold is much greater than the time accessed by these simulations, making it unknown whether the adsorbed proteins were able to reach conformational equilibrium by the end of simulations. On the other hand, for peptides with well-defined secondary structure, and possibly fast-folding proteins, ${ }^{58-60}$ it should be possible to explore conformational equilibria, especially when enhanced sampling techniques are employed. ${ }^{61-64}$ Indeed, recent computational work by Meißner et al. ${ }^{65}$ used enhanced sampling (Hamiltonian replica exchange) to predict circular dichroism spectra for a peptide adsorbed to a model of a silica nanoparticle surface, revealing loss of $\alpha$-helical structure upon adsorption.

In the present work, we focus on graphenic materials and their oxidized derivatives, due to their growing use in research, ${ }^{66-69}$ industry, medicine, and commercial products, $^{70-72}$ as well as the fact that computational models of these materials are well validated. ${ }^{30}$ Here, due to their similar physicochemical properties at the nanoscale, we consider single-layer and multilayer graphene and carbon nanotubes, as well as their hydroxylated derivatives, under the umbrella of graphenic materials. Like other nanomaterials, it has been shown that the formation of a protein corona on graphenic materials determines their cellular uptake, physiological distribution, route of excretion, and toxicology; ${ }^{, 73-75}$ hence, understanding protein interaction with these materials is essential for their safe use in industry and the design of nanomedicine. Molecular simulation is particularly apt for study of these materials, as we have recently shown that the CHARMM molecular dynamics force field ${ }^{76}$ coupled with enhanced sampling methods can yield excellent predictions (correlation coefficient $r \geq 0.9$ ) of relative adsorption affinities of small aromatic compounds with a variety of functional groups on naked and hydroxylated carbon nanotubes. ${ }^{30}$

Although recent work by Hughes et al. ${ }^{48,77}$ has made significant progress in understanding the contribution of different amino acid side chains to peptide binding on graphenic materials, the role of the peptide backbone and the effect of adsorption on the backbone conformational equilibrium has remained unexplored. Here, we seek to leverage molecular dynamics and enhanced sampling by the adaptive biasing force method to elucidate the atomic-scale interactions that underlie peptide backbone interaction with graphenic materials. Furthermore, we determine the influence of hydroxy groups on the affinity of the peptide backbone for the surface and backbone conformation. To study these interactions at the most basic level, we consider as a model peptide the $\alpha$-amino acid L-alanine, capped by an acetyl moiety at its $\mathrm{N}$-terminus and a methyl amide at its C-terminus, also known as alanine dipeptide or Ac-Ala-NHMe. This molecule is to protein structure as the hydrogen atom is to atomic structure, being the smallest molecule that possesses structure similar to the backbone of proteins, excluding glycine, proline, and adjacent residues. Ac-Ala-NHMe has a long history of use in understanding backbone conformational preferences, ${ }^{78-82}$ although protein backbone dynamics are not well represented by this model molecule. ${ }^{83}$ The Ramachandran plot $^{84}$ for AcAla-NHMe, showing the joint preferences for $\phi$ and $\psi$ dihedral angles, reveals favorable regions corresponding to the backbone structures like those in $\alpha$-helices and $\beta$-sheets. Here we study the effect of naked and hydroxylated graphenic carbon on the Ramachandran free-energy landscape (the potential of mean force as a function of $\phi$ and $\psi$ torsional angles), quantifying the influence of the distance from the graphenic surface and any covalently bound hydroxy moieties.

\section{METHODS}

Molecular Models. Model generation began with a single alanine amino acid. The $\mathrm{N}$-terminus was conjugated with an 


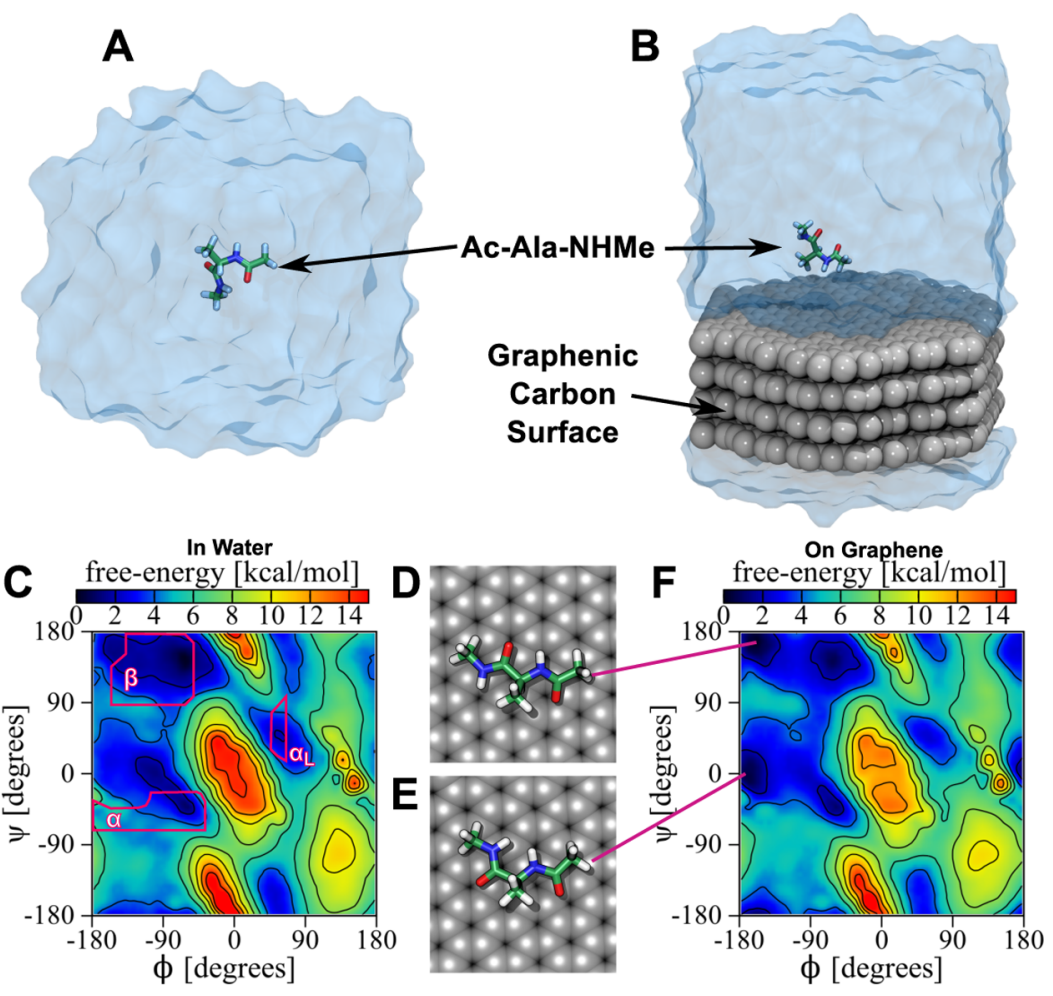

Figure 1. Conformational free-energy landscapes of Ac-Ala-NHMe in bulk water and at the graphene-water interface. Molecular dynamics models of Ac-Ala-NHMe in water (A) and at the graphene-water interface (B). Both systems are periodic along all three axes; thus, the system shown in panel B has an effectively infinite graphene surface. The Ac-Ala-NHMe molecules are shown with a ball-and-stick representation with $\mathrm{H}, \mathrm{C}, \mathrm{N}$, and $\mathrm{O}$ atoms in white, green, blue, and red, respectively. The carbon atoms of the four graphene layers are shown as gray spheres. Although the systems contained explicit water molecules, they are represented here by a translucent cyan surface for clarity. (C) Ramachandran free-energy landscape for Ac-Ala-NHMe in water. Regions outlined in magenta and denoted $\alpha, \beta$, and $\alpha_{\mathrm{L}}$ are associated with right-handed $\alpha$-helices, $\beta$-sheet, and left-handed $\alpha$-helices, respectively. (D) Representative conformation of Ac-Ala-NHMe corresponding to the global free-energy minimum in panel $\mathrm{F}$ (here called $\beta$-planar). (E) Representative conformation of Ac-Ala-NHMe corresponding to the second-lowest local free-energy minimum in panel $\mathrm{F}$ (here called $\alpha$-planar). (F) Ramachandran free-energy landscape for Ac-Ala-NHMe at the graphene-water interface. The plots are anchored such that the minimum free energy is zero.

acetyl group (Ac) and the C-terminus with an NHMe group, using standard patches in the CHARMM 36 force field. ${ }^{85,86}$ The CHARMM36 parameters include tables of potential values for $\phi$ and $\psi$ dihedral crossterms (CMAP) that produce a more realistic free-energy landscape on $(\phi, \psi){ }^{87}$ The resulting single molecule of Ac-Ala-NHMe was immersed in a cubic box of 445 standard TIP3P water molecules. Figure $1 \mathrm{~A}$ shows the final system with 1357 atoms, contained in a periodic box of $25 \times 25$ $\times 25 \AA^{3}$. Additionally, to explore the role of the chemical nature of the backbone on its interaction with the graphene surface, we also simulated butanone, methyl acetate, and $\mathrm{N}$-methylacetamide. These molecules were extracted for the MolView database $^{88}$ and parametrized using the ParamChem web interface (CHARMM General Force Field version 3.0.1). ${ }^{89,90}$ To evaluate the free energy of adsorption of Ac-Ala-NHMe to graphenic surfaces, we used two models of naked multilayer graphenic carbon. The graphenic surface was represented using the standard benzene-like aromatic carbon type (CG2R61) of the CHARMM general force field. We considered using the GRAPPA force field described by Hughes et al. ${ }^{77}$ however, as shown in Figure S1 in the Supporting Information, GRAPPA did not perform as well as the standard parameters when compared to experimental adsorption data for small aromatics on large-diameter carbon nanotubes. ${ }^{30}$ The parameters for the hydroxylated surfaces were based on a CHARMM general force field model of tert-butanol as detailed in Comer et al..$^{30} \mathrm{~A}$ full set of files for reproducing a free-energy calculation, including these parameters, is included in the Supporting Information. Figure 1B shows the first surface, previously published by Comer et al., ${ }^{30}$ which is composed of four layers of graphene in a graphite arrangement, in which each layer was a supercell of the graphene lattice, in the form of a regular hexagon. After equilibration using the barostat (Langevin piston) parameters described further below, the hexagonal side length averaged about $16.95 \AA$, which is equivalent to a parallelogram unit cell with basis vectors $\langle a, 0,0\rangle$ and $\left\langle a / 2, a(3)^{1 / 2} / 2,0\right\rangle$, for $a=$ $29.35 \AA$. The layers were periodically continued throughout in the $x y$ plane, forming an effectively infinite flat surface. ${ }^{30}$ Figure $1 \mathrm{~B}$ shows the final simulation system after solvated with 758 water molecules of the standard TIP3P water model of CHARMM, reaching a total of 3448 atoms. To increase the computational efficiency of some simulations (such as those to obtain three-dimensional potentials of mean force, described below), a second smaller system was created. The model shown in Figure S2A of the Supporting Information was built using two layers of graphene in a rectangle of $19.6 \AA \times 21.2 \AA$. Figure S2A shows the final rectangular system after being solvated with 292 TIP3P water molecules, giving a total of 1218 atoms. Figure S2B shows that the 3448-and 1218-atom systems yield statistically indistinguishable results for a calculation of the free energy as a function of the distance between the first layer graphene and Ac-Ala-NHMe. Additionally, we used two 
hydroxylated graphene surfaces described in Comer et al. ${ }^{30}$ Figure 4B,D shows the different arrangements of hydroxy groups on the hexagonal multilayer graphene model described above. The model in Figure 4B contains 9 uniformly distributed hydroxy groups (graph-OH(1)), while the model shown in Figure 4D possesses 18 uniformly spaced hydroxy groups (graph-OH(2)). ${ }^{30}$ Two additional surface models were created by covalently conjugating a single hydroxy group or pair of hydroxy groups to the small rectangular graphene system, illustrated in Figure 6. Manipulation of the molecular systems, as well as visualization, analysis, and rendering, was accomplished using VMD $1.9 .2^{91}$ and the associated plugin TopoTools. $^{92}$

Molecular Dynamics Simulation. All simulations were executed in the molecular dynamics software NAMD $2.11^{93}$ applying the all-atom CHARMM36 force field. ${ }^{85,86}$ Using the Langevin thermostat and Langevin piston ${ }^{94}$ method, we maintained the temperature and pressure at $300 \mathrm{~K}$ and $101.325 \mathrm{kPa}(1.0 \mathrm{~atm})$, respectively. The Langevin piston algorithm was free to equilibrate the size of the hexagonal system along the $z$ axis and in the $x y$ plane independently (while maintaining a regular hexagon in the $x y$ plane). The size of the rectangular system was equlibrated along each of the three axes independently. For convenience, the atoms of the layers of graphene not making contact with Ac-Ala-NHMe were restrained to their initial positions along the $z$-axis by harmonic restraints with force constants of $\kappa=1 \mathrm{kcal} \mathrm{mol}^{-1} \AA^{-1}, \mathbf{F}_{\text {rest }}=$ $-\kappa\left(z_{i}-z_{i}^{0}\right) \hat{\mathbf{z}}$. No restraints were applied to the graphene layer making contact with Ac-Ala-NHMe, nor were restraints with nonzero components in the $x y$ plane applied, allowing lateral relaxation of the graphene under the Langevin piston parameters described above. A smooth 8-9 A cutoff of van der Waals forces was employed (see the Supporting Information of Comer et al. ${ }^{30}$ for justification of this cutoff scheme). The particle-mesh Ewald algorithm ${ }^{95}$ was used to compute the electrostatic interactions with a mesh spacing of $<1.2 \AA$. The lengths of covalent bonds involving hydrogen atoms were constrained ${ }^{96,97}$ to the values prescribed by the CHARMM force field. The masses of nonwater hydrogen atoms were increased by a factor of 3 (to $3.0240 \mathrm{Da}$ ) by transferring mass from the heavy atom to which they were attached, allowing us to integrate the equations of motion with a time step of $4 \mathrm{fs}$ with no appreciable loss of accuracy in thermodynamic quantities ${ }^{98}$ (see Figure S3 for corroboration). All systems shown in this work underwent 15,000 steps of energy minimization followed by $10 \mathrm{~ns}$ of equilibration before beginning at least $400 \mathrm{~ns}$ of simulation using free-energy calculation methods, described below. A portion of the simulations made use of Extreme Science and Engineering Discovery Environment (XSEDE) resources. ${ }^{99}$

Free Energy in $(\phi, \psi)$. We used the adaptive biasing force $^{100-102}(\mathrm{ABF})$ method to calculate the Ramachandran free-energy landscape, ${ }^{103}$ over the two dihedral angles $\phi, \psi$ of the Ac-Ala-NHMe backbone. The transition coordinates $\phi$ and $\psi$ were the dihedral torsional angles formed respectively by the $\mathrm{N}-\mathrm{C}_{\alpha}-\mathrm{C}-\mathrm{N}$ and $\mathrm{C}-\mathrm{C}_{\alpha}-\mathrm{N}-\mathrm{C}$ atoms of Ac-Ala-NHMe. Both transition coordinates were implemented through the Colvars module, ${ }^{104}$ exploring the full range of rotation $\left(-180\right.$ to $\left.180^{\circ}\right)$ of each dihedral, with a bin width for $\mathrm{ABF}$ samples of $5.0^{\circ}$. The two-dimensional (2D) potential of mean force (PMF), $w_{2 \mathrm{D}}(\phi$, $\psi)$, was calculated for Ac-Ala-NHMe in an aqueous environment (400 ns), on the hexagonal graphenic system (323 ns), and on both hydroxylated graphene surfaces (400 ns each).
Although adsorption of Ac-Ala-NHMe to the graphene surfaces is highly favored, spontaneous desorption of Ac-Ala-NHMe and diffusion into the aqueous medium was sometimes observed. While this was a rare event with little effect on the resulting free energies, a harmonic wall was applied for distances between the center of mass of the peptide and the first graphene layer $Z>$ $10 \AA$. Convergence of this free-energy calculation is analyzed in Figure S4 of the Supporting Information, which shows steady convergence of the estimated mean force after about 40 ns of simulated time.

Free Energy in Z. To calculate the free energy of adsorption of Ac-Ala-NHMe to the graphenic surface, we defined $Z$ as the distance along the $z$-axis of the center of mass of Ac-Ala-NHMe from the center of mass of the first layer of graphene and calculated the PMF $w_{1 \mathrm{D}}(Z)$. The $z$-axis is orthogonal to the plane of the graphene. $A B F$ was used to calculate the PMF using a single window on the domain $Z \in[3$, 10] $\AA$, with a bin width of $\Delta Z=0.05 \AA$. The calculations for the hexagonal and rectangular systems (see Figure S2 of the Supporting Information) had durations of 600 and $638 \mathrm{~ns}$, respectively. The error bars in Figure $3 \mathrm{~F}$ are computed as described in the Supporting Information.

Free Energy in $(\phi, \psi, Z)$. To obtain a better understanding of the adsorption of Ac-Ala-NHMe to naked graphenic surfaces, we used the $A B F$ method simultaneously on the three transition coordinates $\phi, \psi$, and $Z$ previously described (see the Supporting Information for the Colvars ${ }^{104}$ configuration file). Because of the large number of bins in the threedimensional (3D) calculation, a coarser grid was used, with bin widths along $\phi, \psi$, and $Z$ being $10^{\circ}, 10^{\circ}$, and $0.15 \AA$, respectively. The range of the transition coordinates was the same as in previous calculations, using a single window. The total simulation time was 1093 ns. The 1D PMF along $Z$ was derived from this by applying the following equation

$$
w_{3 \mathrm{D}}^{1 \mathrm{D}}(Z)=-k_{\mathrm{B}} T \ln \left\{\int \mathrm{d} \phi \int \mathrm{d} \psi \exp \left(-\beta w_{3 \mathrm{D}}(\phi, \psi, Z)\right\}\right.
$$

where $w_{3 \mathrm{D}}(\phi, \psi, Z)$ is the full $3 \mathrm{D}$ PMF and $\beta$ is the inverse of the thermal energy, e.g., $\beta=1 / k_{\mathrm{B}} T$. Figure $3 \mathrm{E}$ characterizes the similarity between cross sections of $w_{3 \mathrm{D}}(\phi, \psi, Z)$ and the reference Ramachandran free-energy landscape of Ac-Ala$\mathrm{NHMe}$ in bulk water. The root-mean-square difference, $\delta F\left(Z_{k}\right)$, between the cross section at $Z_{k}$ and the reference was calculated according to

$$
\begin{aligned}
\delta F\left(Z_{k}\right)^{2}= & \sum_{i, j}\left\{\left[\partial_{\phi} w_{3 \mathrm{D}}\left(\phi_{i}, \psi_{j}, Z_{k}\right)-\partial_{\phi} w_{2 \mathrm{D}}^{\text {wat }}\left(\phi_{i}, \psi_{j}\right)\right]^{2}\right. \\
& \left.+\left[\partial_{\psi} w_{3 \mathrm{D}}\left(\phi_{i}, \psi_{j}, Z_{k}\right)-\partial_{\psi} w_{2 \mathrm{D}}^{\text {wat }}\left(\phi_{i}, \psi_{j}\right)\right]^{2}\right\}
\end{aligned}
$$

To avoid defining a reference potential, we compared the derivatives of the potentials of mean force (equivalent to the negative of the thermodynamic mean force), rather than the potentials themselves. The convergence of this calculation can be inferred from Figure $3 \mathrm{E}$.

Free Energy in $(\phi, \psi, d)$. To determine the effect of the surface hydroxy groups on the conformational free-energy landscape of Ac-Ala-NHMe, we performed further 3D ABF calculations on the transition coordinates $\phi, \psi$, and $d$, the latter being defined as the distance between the center of mass of the $\mathrm{OH}$ groups and the center of mass of Ac-Ala-NHMe projected onto the $x y$ plane. As is shown in Figure 6A, the simulations were carried out on two square surfaces composed of four 
layers of graphenic carbon. The first system had a single covalently attached $\mathrm{OH}$ group and the second system, a pair of $\mathrm{OH}$ groups. Both models were solvated with 914 water molecules, reaching a final size of $30 \times 30 \times 50 \AA^{3}$. The dihedrals $\phi$ and $\psi$ were explored in the full range of rotation of -180 to $180^{\circ}$ with a bin width of $10.0^{\circ}$; on the other hand, the distance $d$ was evaluated from 0 to $8.5 \AA$ with a bin width of $0.15 \AA$. The single $\mathrm{OH}$ and $\mathrm{OH}$ pair systems were equilibrated with the protocol described above, followed by the use of the ABF method for 3932 and 3388 ns, respectively. In the plots, Figure $6 \mathrm{C}, \mathrm{D}$, the geometric contribution to the free energy $\left(W_{\text {geo }}=-k_{\mathrm{B}} T \ln (2 \pi d)\right)$ was subtracted out, so that the curves would be flat at large distances from the $\mathrm{OH}$ groups. The PMFs were anchored so that the global minimum was zero.

Unbiased Simulations in $\boldsymbol{\beta}$-Planar and $\boldsymbol{\beta}$ Conformations. To carefully study interactions among the solute, surface, and solvent, we performed simulations of Ac-Ala-NHMe in conformations associated with $\beta$ and $\beta$-planar free-energy minima. To prevent transitions out of the $\beta$ conformation, flat bottom harmonic potentials were applied to restrain the $\phi$ and $\psi$ dihedrals to the intervals $[-68,-48]$ and $[-133,-153]$, respectively. Similarly, for the $\beta$-planar conformation, the $\phi$ and $\psi$ intervals were $[-168,-148]$ and $[-178,-158]$. Other than the application of these restraints, the simulations were unbiased. These simulations were performed for the hexagonal naked graphene system and hydroxylated system graph$\mathrm{OH}(1)$. The total simulated time for the $\beta$ and $\beta$-planar conformations on naked graphene was 327 and 329 ns, respectively. For the hydroxylated graphene, these were respectively 360 and 362 ns.

\section{RESULTS AND DISCUSSION}

We first compare the Ramachandran free-energy landscape for Ac-Ala-NHMe in bulk water and on the graphenic surface, identifying considerable differences in the most favorable conformations. We then discuss the role of amide $-\pi$ interactions and interfacial water in determining the Ac-Ala$\mathrm{NHMe}$ conformational ensemble on a graphenic surface. Finally, we consider the influence of hydroxylation of the surface on the conformational thermodynamics of Ac-AlaNHMe.

Ramachandran Landscape in Water and on Naked Graphene. First, we compared the potential of mean force (PMF) as a function of the $\phi$ and $\psi$ dihedral angles ${ }^{80}$ for AcAla-NHMe (alanine dipeptide) in two environments, namely, solvated in water or adsorbed at the graphene-water interface. Images of the simulation systems are shown in Figure 1A,B. The model of the graphene surface shown in Figure $1 \mathrm{~B}$ serves an accurate representation of multilayer graphene and multiwall carbon nanotubes of sufficiently large diameters, ${ }^{29,30}$ as well as an approximation of single-layer graphene and large singlewalled nanotubes (the effect of the number of layers on the adsorption free energy of small neutral molecules was shown to be slight in the Supporting Information of Comer et al. ${ }^{30}$ ). Figure 1C shows the Ramachandran free-energy landscape of Ac-Ala-NHMe in bulk water using the newest version of the CHARMM force field for proteins, CHARMM36. ${ }^{86}$ In agreement with experiment and quantum chemistry calculations, ${ }^{82}$ this force field gives a $\beta$-sheet-like structure as the global minimum and a somewhat less favorable secondary minimum, corresponding to the right-handed $\alpha$-helix. In the calculation, the global free-energy minimum appears at $(\phi, \psi)=$ $\left(-58^{\circ}, 143^{\circ}\right)$ near the right edge of the region denoted $\beta$ and is similar to the backbone conformation of the polyproline II helix found in the collagen, while the second most favorable minimum, at $\left(-63^{\circ},-38^{\circ}\right)$, coincides precisely with the canonical- $\alpha$ helix. The landscape in Figure $1 \mathrm{C}$ has some quantitative differences with those calculations using older force fields; ${ }^{79,80,105}$ however, our calculation using the previous version of the CHARMM force field CHARMM22/CMAP, ${ }^{87}$ shown in Figure S5 of the Supporting Information, quantitatively agrees with a similar calculation by Esque and Cecchini. ${ }^{106}$

A comparison of parts $\mathrm{C}$ and $\mathrm{F}$ of Figure 1 reveals the effect of the graphenic surface on the Ramachandran free-energy landscape. Most strikingly, the global free-energy minimum is displaced from the $\beta$-sheet-like region to a more planar structure corresponding to $\left(-158^{\circ},-168^{\circ}\right)$. This structure, which is illustrated in Figure 1D, is referred to here as $\beta$-planar. Likewise, a new distinct minimum appears at $\left(-173^{\circ},-3^{\circ}\right)$, corresponding to a planar version of the $\alpha$-helix-like structure, here called $\alpha$-planar and illustrated in Figure 1E. Thus, the $\beta$ planar and $\alpha$-planar structures are dominant on the graphene surface, while the $\beta$ and $\alpha$ structures retain some prevalence, being associated with minimum free energies $<1 \mathrm{kcal} / \mathrm{mol}$ from the $\beta$-planar global minimum. The minima of the Ramachandran free-energy landscapes in all environments considered in this work are summarized in Table 1 . It might be noted that a structure apparently similar to $\beta$-planar was seen by Chipot and Pohorille $^{80}$ at a hexane-water interface.

Table 1. Major Free-Energy Minima in Ramachandran FreeEnergy Landscapes for Ac-Ala-NHMe ${ }^{a}$

\begin{tabular}{llrrl}
\hline \multicolumn{1}{c}{ system } & region & \multicolumn{1}{c}{$\phi$} & \multicolumn{1}{c}{$\psi$} & free energy \\
\hline water & $\beta$ & -58 & 143 & 0 \\
water & $\beta$-planar & -148 & 158 & $1.3 \pm 0.1$ \\
water & $\alpha$ & -63 & -38 & $0.8 \pm 0.2$ \\
water & $\alpha$-planar & & & $\mathrm{N} / \mathrm{A}$ \\
water & $\alpha_{\mathrm{L}}$ & 68 & 43 & $1.8 \pm 0.1$ \\
graph & $\beta$ & -63 & 143 & $0.6 \pm 0.1$ \\
graph & $\beta$-planar & -168 & 168 & 0 \\
graph & $\alpha$ & -58 & -43 & $0.7 \pm 0.1$ \\
graph & $\alpha$-planar & -173 & -3 & $0.3 \pm 0.1$ \\
graph & $\alpha \mathrm{L}$ & 58 & 43 & $1.8 \pm 0.1$ \\
graph-OH(1) & $\beta$ & -58 & 148 & $0.8 \pm 0.1$ \\
graph-OH(1) & $\beta$-planar & -158 & 168 & 0 \\
graph-OH(1) & $\alpha$ & -58 & -43 & $1.5 \pm 0.2$ \\
graph-OH(1) & $\alpha$-planar & -168 & -3 & $1.0 \pm 0.1$ \\
graph-OH(1) & $\alpha_{\mathrm{L}}$ & 63 & 48 & $2.7 \pm 0.1$ \\
graph-OH(2) & $\beta$ & -63 & 143 & 0 \\
graph-OH(2) & $\beta$-planar & -163 & 163 & $1.1 \pm 0.2$ \\
graph-OH(2) & $\alpha$ & -58 & -43 & $1.0 \pm 0.2$ \\
graph-OH(2) & $\alpha$-planar & -168 & -8 & $0.7 \pm 0.2$ \\
graph-OH(2) & $\alpha$ L & 63 & 48 & $2.0 \pm 0.3$ \\
\hline
\end{tabular}

${ }^{a} \phi$ and $\psi$ angles are in units of deg, and free energies are in $\mathrm{kcal} / \mathrm{mol}$. Each Ramachandran landscape is anchored so that the minimum value is zero.

The Role of Amide $-\pi$ Interaction. Amide $-\pi$ interactions, typically involving contacts between amides and aromatic moieties, are energetically favorable in the $\mathrm{NH}$ face-on geometry, where the $\mathrm{NH}$ bond is orthogonal to the plane of the aromatic ring and directed toward its center, as well as in the stacked geometry, where the entire amide lies in a plane parallel to that of the aromatic ring. ${ }^{107}$ Our results suggest that 


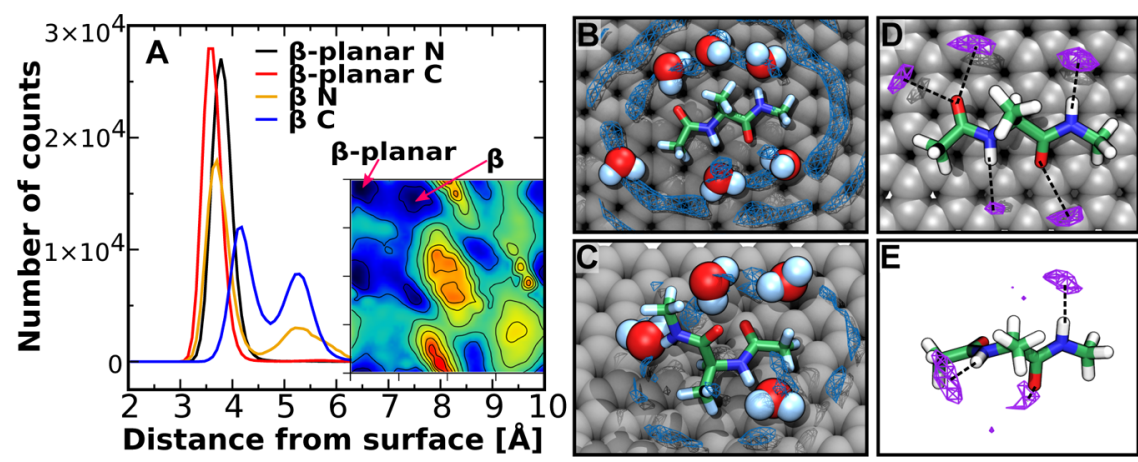

Figure 2. Contact between the graphenic surface and the amide groups of Ac-Ala-NHMe. (A) Histograms of the distance between the first layer of graphene and the nitrogen atom or carbon atom on either side of the $\mathrm{C}_{\alpha}$ atom of Ac-Ala-NHMe. The results were obtained from simulations in which Ac-Ala-NHMe remained in the vicinity of either the $\beta$-planar or $\beta$ free-energy wells, indicated in the inset (see Methods). (B) Representative snapshot of simulations in which Ac-Ala-NHMe adopts a $\beta$-planar conformation at the graphene-water interface. Water molecules near the Ac-AlaNHMe molecule are explicitly shown. The blue wireframes represent enclose positions relative to the Ac-Ala-NHMe molecule where the average water density is $>2.7 \mathrm{~g} / \mathrm{mL}$. (C) Representative snapshot of simulations in which Ac-Ala-NHMe adopts a $\beta$ conformation at the graphene-water interface. Blue wireframes have the same meaning as in panel B. (D, E) Regions of very high water density near Ac-Ala-NHMe in its most favorable conformations on graphene (D) and in water (E). The violet wireframes represent regions of average water density $>5.4 \mathrm{~g} / \mathrm{mL}$. Dotted black lines represent hydrogen bonds formed when water molecules occupy these regions.

the favorability of the $\beta$-planar and $\alpha$-planar structures on graphenic carbon emerges from amide $-\pi$ interactions between the Ac-Ala-NHMe backbone and graphene in the stacked configuration. To better analyze the configuration of Ac-AlaNHMe relative to the graphene surface, we performed simulations of Ac-Ala-NHMe in which the conformations were kept within the $\beta$ and $\beta$-planar free-energy basins (see Methods). Figure 2A shows histograms of the distance between the graphene surface and the nitrogen atom or carbon atom adjacent to the $\mathrm{C}_{\alpha}$ atom of Ac-Ala-NHMe for the $\beta$ and $\beta$ planar conformations. The minimum free-energy of the $\beta$ planar conformation is clearly associated with both amide groups of Ac-Ala-NHMe making close contact with the graphene surface, as demonstrated by the black and red curves in Figure 2A and exemplified in Figure 2B. On the other hand, the inherent lack of planarity of the $\beta$ conformation implies that only one of the two amide groups can make contact with a flat graphene surface at any point in time. The propensities of these two amide groups to stack on the surface surface are not equivalent, as demonstrated in Figure 2A. The orange and blue curves in this figure indicate that, in the $\beta$ conformation, there is a high propensity for the $\mathrm{N}$-terminal amide to lie flat on the surface, while the C-terminal amide has an oblique conformation, illustrated in Figure 2C. Interestingly, in this $\beta$ conformation, two well-defined peaks appear in the histogram for the C-terminal carbon atom (blue curve). As demonstrated by Figure S6 of the Supporting Information, we find that the higher peak at $Z=4.2 \AA$ corresponds to the $\mathrm{NH}$ group of the $\mathrm{C}$-terminal amide adopting the favorable $\mathrm{NH}$ face-on geometry ${ }^{107}$ with the graphene surface. For the second peak at $Z=5.3 \AA$, the $\mathrm{NH}$ group does not make direct contact with the graphene but instead forms a hydrogen bond with an interfacial water molecule. The latter configuration is visible in Figure 2C.

To better understand why the free energy of the $\beta$-planar conformation on graphene is less than that of the $\beta$ conformation, we attempted to distinguish various contributions to the free energy for the simulations in these two conformations. First, averaging the total potential energy of the simulation systems, we find that the $\beta$-planar system has a lower potential energy, $\Delta U_{\beta \rightarrow \beta \mathrm{p}}=-0.92 \pm 0.11 \mathrm{kcal} / \mathrm{mol}$, which is mostly due to favorable van der Waals interactions, $\Delta U_{\beta \rightarrow \beta p}^{\mathrm{vdW}}=$ $-2.44 \pm 0.09 \mathrm{kcal} / \mathrm{mol}$. This favorable change in the van der Waals energy is opposed by the greater mechanical strain in the $\beta$-planar conformation than in the $\beta$ conformation. The $\beta$ and $\beta$-planar conformations have average potential energies in the force field's bonded terms of $18.34 \pm 0.03$ and $19.79 \pm 0.03$ $\mathrm{kcal} / \mathrm{mol}$, respectively, giving $\Delta U_{\beta \rightarrow \beta p}^{\text {bonded }}=1.45 \pm 0.04 \mathrm{kcal} / \mathrm{mol}$. A small unfavorable change in electrostatic energy is also observed. The fact that the change in internal energy is more favorable than the change in free energy $\left(\Delta U_{\beta \rightarrow \beta \mathrm{p}}<\Delta G_{\beta \rightarrow \beta \mathrm{p}}\right)$ suggests that the $\beta$-planar conformation is less favorable in terms of entropy, $T \Delta S_{\beta \rightarrow \beta \mathrm{p}} \approx 0.3 \mathrm{kcal} / \mathrm{mol}$, which may relate to the more ordered solvent configuration for this conformation (see Figure 2B,C). Note that the difference in the mean volume of the simulation system between the two conformational states is $-6.1 \pm 0.5 \AA^{3}$, which corresponds to a pressure-volume work of $\sim 10^{-4} \mathrm{kcal} / \mathrm{mol}$, implying that enthalpy and internal energy are essentially equivalent for this transition, a typical situation for small molecules in water. ${ }^{108,109}$

As further evidence that the strong physisorption between the peptide backbone and graphene can be attributed to amide $-\pi$ interactions, we compare free-energy calculations for the graphene adsorption of a molecule representing the peptide backbone ( $N$-methylacetamide) and its ester analogue (methyl acetate). The results are shown in Figure S7 in the Supporting Information. Although both molecules have similar planar geometries and adopt stacked conformations on the graphene surface, the adsorption free energy of the amide is more favorable than the ester by $-0.66 \pm 0.11$ and $-1.3 \pm 0.2 \mathrm{kcal} /$ mol, for adsorption from aqueous solution and from the gas phase, respectively. These results suggest that the high affinity of the amide for graphene observed in the simulations is not merely a geometric effect but is due to the chemical nature of the amide group as captured by the force field. Notably, in the CHARMM force field, the van der Waals interaction of an aromatic carbon with the amide $\mathrm{N}$ in $\mathrm{N}$-methylacetamide is significantly stronger that of the ester $-\mathrm{O}-$ at the analogous position in methyl acetate.

The Role of Water. The simulations suggest that the graphene surface is coordinated by water molecules, with a density reaching nearly 3 times that of bulk water at a distance 

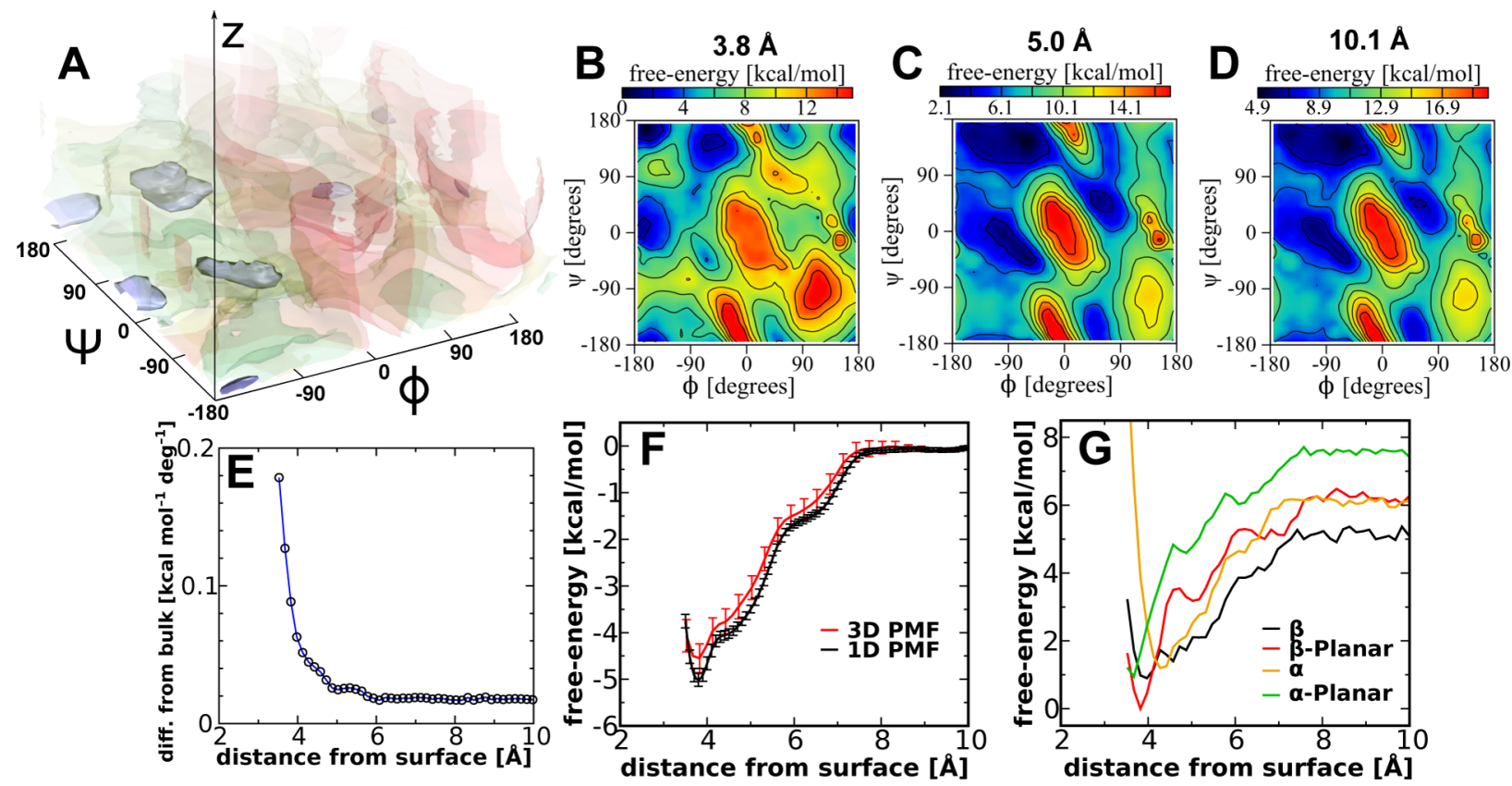

Figure 3. Dependence of the conformational free-energy landscape on the distance from a graphenic surface. (A) Volumetric plot of the threedimensional potential of mean force (3D PMF) of Ac-Ala-NHMe as a function of the $\phi$ and $\psi$ torsions and $Z$, the distance between Ac-Ala-NHMe and the graphene layer. The PMF is anchored such that the minimum free energy is zero. Isosurfaces of free energy at $3,6,9$, and $12 \mathrm{kcal} / \mathrm{mol} \mathrm{shown}$ in blue, green, yellow, and red. (B-D) Cross sections of this PMF at $z$ values of 3.8, 5.0, and 10.1 A. Note that the color scales in panels C and D are adapted to facilitate comparison of the conformational preferences. (E) Convergence of the $(\phi, \psi)$ free-energy landscape of Ac-Ala-NHMe to that in bulk water as the distance from the surface increases. Plotted is the root-mean-square difference between the reference mean force in water (Figure $1 \mathrm{C})$ and $(\phi, \psi)$ cross sections of the 3D mean force. (F) PMF as a function of distance between the graphene layer and Ac-Ala-NHMe obtained from a $1 \mathrm{D}$ ABF calculation or by integrating the 3D ABF result (see Methods). (G) Free energy associated with local minima of the Ramachandran landscape as a function of $Z$.

of $3.3 \AA$ (see Figure S8 of the Supporting Information). In aqueous solution, Ac-Ala-NHMe is also coordinated by water molecules, which form hydrogen bonds with the amide $\mathrm{N}$ and $\mathrm{O}$ atoms. Although adsorption to the graphene surface considerably reduces the solvent-accessible surface area of AcAla-NHMe, the number of hydrogen bonds to water does not change much, from $4.1 \pm 1.9$ (mean \pm SD) in bulk water to 3.9 \pm 1.6 on the graphene surface, owing to the enhancement of the water density at the graphene-water interface. The average number of hydrogen bonds as a function of distance from the surface is shown in Figure S9 of the Supporting Information. The presence of Ac-Ala-NHMe at the interface leads to welldefined solvent structure, particularly in the case of the $\beta$-planar conformation (Figure 2B,C). The greater localization of water for the $\beta$-planar conformation could be responsible for this conformation's greater entropy as compared to the $\beta$ conformation. Although the number of solvent hydrogen bonds for Ac-Ala-NHMe on graphene is slightly less than that for Ac-Ala-NHMe in bulk water, the positions of these water molecules are much better defined on graphene than in the bulk (Figure 2D,E). Figure 2D shows five well-defined locations for hydrogen-bonded water molecules.

Orientation of Ac-Ala-NHMe on Graphene. The reader may have noticed that the $\mathrm{CH}_{3}$ side chain of Ac-Ala-NHMe is oriented away from the surface in all of the images of adsorbed Ac-Ala-NHMe that we have shown so far (Figures 1 and 2). As demonstrated in Figure S10 of the Supporting Information, this orientation is indeed highly favored for distances between AcAla-NHMe and the graphene layer $Z<4 \AA$. The favorability of this orientation can be easily understood because it permits the amide groups to make close contact with the surface without steric interference from the side chain. However, side chains with higher affinities for graphene $\mathrm{e}^{48}$ (Arg, Tyr, Phe) may favor a different orientation. Interestingly, at distances of $5.0 \AA<Z<$ $5.7 \AA$, the $\mathrm{CH}_{3}$ side chain tends to be oriented toward the graphene (Figure $\mathrm{S} 10$ of the Supporting Information). For $Z>$ $7.5 \AA$, the orientation of Ac-Ala-NHMe becomes isotropic.

Three-Dimensional Free-Energy Landscape. The PMF shown in Figure $1 \mathrm{~F}$ represents a thermodynamic average over a range of distances between the Ac-Ala-NHMe molecule and the graphene layer, dominated by the most favorable distances, $Z \in$ $[3.8,4.1] \AA$ (see Figure S11 of the Supporting Information). To better understand the influence of graphene on the Ac-Ala$\mathrm{NHMe}$ structure, we calculated a three-dimensional potential of mean force (3D PMF), including $Z$, the distance between the first graphene layer and the center of mass of Ac-Ala-NHMe as a third transition coordinate, in addition to $(\phi, \psi)$. Figure $3 \mathrm{~A}$ is a volumetric plot of this potential of mean force, showing that the lowest free energy regions appear near the graphene surface and are associated with the $\beta$-planar, $\beta, \alpha$-planar, $\alpha$, and $\alpha_{\mathrm{L}}$ conformations. The cross section through the 3D PMF at $Z=$ $3.85 \AA$, shown in Figure $3 \mathrm{~B}$, passes through the global minimum of the $3 \mathrm{D} \mathrm{PMF}$, exhibiting a free energy landscape dominated by the $\beta$-planar conformation. The $\alpha$-planar and $\beta$ conformations also appear. As might be expected, the cross sections appear more like the Ramachandran PMF in aqueous solution as the distance between the graphene layer and Ac-Ala$\mathrm{NHMe}$ is increased. The cross section at a distance of $Z=10.1$ $\AA$ (Figure 3D) is effectively identical to that in aqueous solution (Figure 1C).

Figure $3 \mathrm{E}$ quantifies the approach to aqueous phase conformational preferences as the distance between Ac-Ala- 

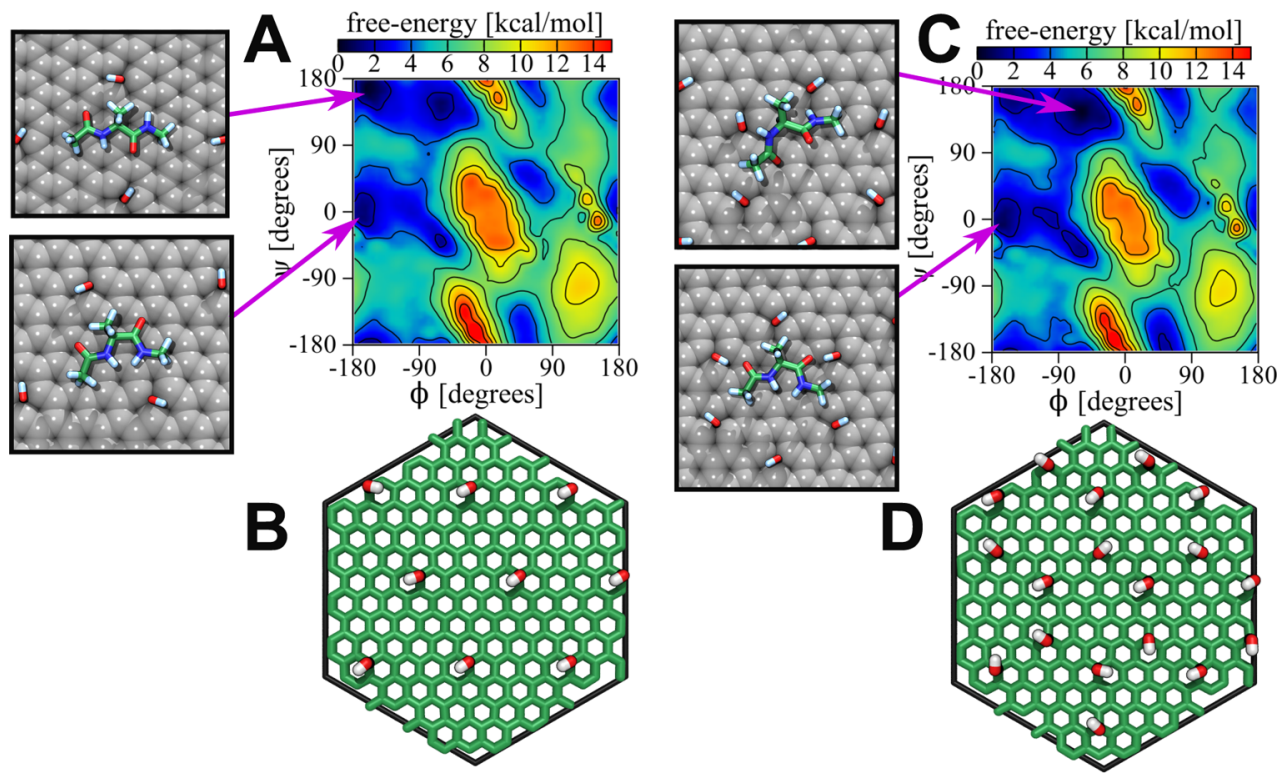

Figure 4. Conformational preferences of Ramachandran free-energy landscapes for Ac-Ala-NHMe adsorbed to two different hydroxylated graphenic surfaces. (A) Ramachandran free-energy landscape of Ac-Ala-NHMe adsorbed to the hydroxylated surface shown in panel B. Representative snapshots of the conformations corresponding to local free-energy minima are indicated by arrows. (B) Top view of a hydroxylated graphene surface model, containing 9 uniformly distributed $\mathrm{OH}$ groups (graph-OH(1)). (C) Ramachandran free-energy landscape of Ac-Ala-NHMe adsorbed to the hydroxylated surface shown in panel D. (D) Top view of a hydroxylated graphene surface model, containing 18 uniformly distributed $\mathrm{OH}$ groups (graph-OH(2)).

NHMe and surface increases. While the Ramachandran freeenergy landscape of Ac-Ala-NHMe near the graphene-water interface is considerably different from that in the bulk (Figure $3 \mathrm{~B})$, it converges to that in bulk water by $Z \approx 6 \AA$. The fact that the backbone orients away from the surface for $Z \approx 5 \AA$ (demonstrated in Figure S10 of the Supporting Information) may contribute to the rapid loss of the surface's influence on the backbone conformation with increasing $Z$. Figure $3 \mathrm{~F}$ shows the $1 \mathrm{D}$ PMF along $Z$, as computed by numerically integrating the 3D PMF (see Methods). To obtain sufficient sampling, the grid used for the 3D PMF must be rather coarse; thus, we computed a similar PMF along $Z$ in a $1 \mathrm{D}$ ABF calculation at higher resolution, also shown in Figure 3F, which is statistically consistent with that from the $3 \mathrm{D}$ PMF. In both cases, the minimum free energies appear near a distance of 3.8 A between the center of mass of Ac-Ala-NHMe and the graphene layer, corresponding to estimates of the adsorption free energy of $-4.6 \pm 0.3$ and $-5.01 \pm 0.13 \mathrm{kcal} / \mathrm{mol}$, for $1 \mathrm{D}$ and integrated$3 \mathrm{D}$ calculations, respectively. Interestingly, the influence of graphene on the backbone conformation of Ac-Ala-NHMe appears quite short-ranged (Figure 3E), involving direct contact, while the force between the surface and the center of mass of Ac-Ala-NHMe is longer-ranged and remains significant up to at least $Z \approx 8 \AA$ (Figure $3 F$ ), owing to solvent mediated effects.

Figure 3G plots the depth of the free-energy minima associated with $\beta, \beta$-planar, $\alpha$, and $\alpha$-planar conformations in $(\phi, \psi)$ cross sections of the 3D PMF. The relative free energies of the conformations vary considerably as a function of $Z$. For very short distances $Z<3.6 \AA$, the $\alpha$-planar conformation has the lowest free energy, while the nonplanar $\alpha$ and $\beta$ conformations are highly disfavored owing to steric clashes with surface atoms. On the interval $3.6 \AA<Z<4.1 \AA$, the $\beta$ planar conformation becomes the most favored, coinciding with the minimum free energy of the entire 3D PMF, while the $\beta$ conformation becomes the second most likely. The planar conformations rapidly become unfavorable as the distance increases, while the $\alpha$ and $\beta$ conformations have similar free energies on the interval $4.1 \AA<Z<4.7 \AA$. Beyond $Z \approx 4.7 \AA$, the relative free energies of the conformations become similar to that in bulk water, with the $\beta$ and $\alpha$ conformations being the most favored and the $\alpha$-planar minimum becoming indistinct.

Ramachandran Landscape on Hydroxylated Graphene. Oxygen containing defects, including hydroxy groups, are common in graphene produced by thermal reduction of graphene oxide. ${ }^{10-112}$ In previous work, we validated simple models of hydroxylated carbon nanotubes against experimentally derived adsorption free energies, ${ }^{30}$ motivating further exploration of hydroxylated graphenic surfaces. The case of hydroxylated graphenic surfaces is more complex due to the lack of information on the spatial arrangement of $\mathrm{OH}$ groups on the nanotube surface. Here we have used two models of hydroxylated carbon nanotubes previously published by Comer et $\mathrm{al}^{30}$

The first model, referred to here as graph- $\mathrm{OH}(1)$ and having the $\mathrm{OH}$ arrangement shown in Figure $4 \mathrm{~B}$, has a relatively low surface density of $\mathrm{OH}$ groups, $1.2 \mathrm{~nm}^{-1}$. As might be expected, this surface has a Ramachandran free energy landscape (Figure 4A) with many similarities to that on naked graphene. The $\beta$-planar conformation, which is the most favorable on naked graphene, is even more dominant on graph-OH(1), as detailed in Table 1 . The second most favorable conformation on naked graphene, the $\alpha$-planar conformation, is edged out by the $\beta$ conformation on graph$\mathrm{OH}(1)$.

Model graph-OH(2), shown in Figure 4D, has double the $\mathrm{OH}$ density of the first, $2.4 \mathrm{~nm}^{-1}$. The Ramachandran freeenergy landscape (Figure 4D) appears less like that on naked graphene and more similar to that in bulk water. As can be seen in Table 1, the $\beta$ conformation becomes dominant, while the $\beta$ - 
planar conformation is significantly weakened compared to naked graphene and only slightly more favorable on the graph$\mathrm{OH}(2)$ surface than in bulk water. One notable difference between the free-energy landscape on graph-OH(2) and that in bulk water is that the $\alpha$-planar conformation is distinct and more favorable than the $\beta$-planar conformation, which appears to be due to a fortuitous fit of the $\alpha$-planar structure within the $\mathrm{OH}$ arrangement, permitting hydrogen bonds between the $\mathrm{OH}$ groups and the carbonyl oxygen atoms of Ac-Ala-NHMe (Figure 4C, lower simulation snapshot).

Amide- $\pi$ Interaction on Hydroxylated Surfaces. Figure 5 details the effect of interactions of Ac-Ala-NHMe

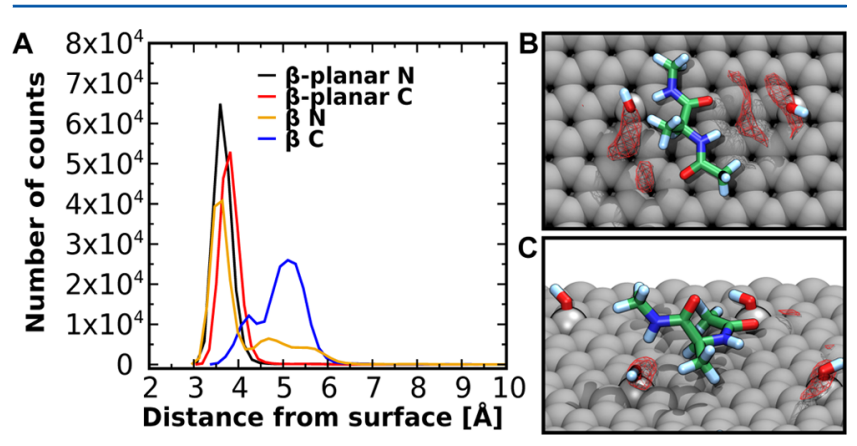

Figure 5. Contact between a hydroxylated graphene surface and the amide groups of Ac-Ala-NHMe. The $\mathrm{OH}$ arrangement for this figure is shown in Figure 4B (graph-OH(1)). (A) Histograms of the distance between the alanine $\mathrm{N}$ - and C-termini and the first layer of hydroxylated graphene for two different Ac-Ala-NHMe conformations. (B) Representative snapshot of Ac-Ala-NHMe in the $\beta$-planar conformation at the hydroxylated graphene-water interface. (C) Representative snapshot of Ac-Ala-NHMe in the $\beta$ conformation at the hydroxylated graphene-water interface. Red wireframes in panels $\mathrm{B}$ and $\mathrm{C}$ enclose positions relative to the Ac-Ala-NHMe molecule at which the average density of surface-bound $\mathrm{OH}$ groups exceeds 70 $\mathrm{mol} / \mathrm{L}$. The snapshots represent only one of the several preferred AcAla-NHMe:OH configurations indicated by these wireframes.

with the $\mathrm{OH}$ groups of the graph-OH(1) surface, using the results of simulations in which Ac-Ala-NHMe was maintained near the $\beta$ and $\beta$-planar free-energy wells (see Methods). As for naked graphene, the $\beta$-planar conformation is associated with both amides making contact with the graphene surface, while the $\beta$ conformation favors contact between the $\mathrm{N}$-terminal amide and the surface, with the C-terminal amide extending away from the surface (Figure 5A). On the other hand, the distribution of the position of this C-terminal amide is markedly different than that for naked graphene. On the graph-OH(1) surface, the most likely configuration of Ac-Ala$\mathrm{NHMe}$ in the $\beta$ conformation corresponds to hydrogen bonding between the C-terminal $\mathrm{NH}$ group and surface bound $\mathrm{OH}$ groups, which is clearly visible in Figure $5 \mathrm{C}$. The histogram peak associated with this configuration, at $Z=5.1 \AA$, appears slightly nearer to the surface than the second peak observed for naked graphene (Figure 2A), because the covalently attached $\mathrm{OH}$ groups lie closer to the plane of the graphene than the interfacial water responsible for the second peak on naked graphene. The peak at $Z=4.2 \AA$, corresponding to direct interaction between the $\mathrm{C}$-terminal $\mathrm{NH}$ group and graphene, which is the most prominent $\beta$ configuration on naked graphene (Figure 2A), is significantly less pronounced in Figure 5A.
The red wireframes in Figure 5B represent positions relative to Ac-Ala-NHMe of high average surface $\mathrm{OH}$ density, and demonstrate that there is a high propensity for hydrogen bonding between surface $\mathrm{OH}$ groups and the amides of Ac-Ala$\mathrm{NHMe}$ in the $\beta$-planar conformation. While the snapshot in Figure $5 \mathrm{~B}$ shows a hydrogen bond with the C-terminal $\mathrm{NH}$ group, the average densities indicate that configurations with a hydrogen bond between a surface-bound $\mathrm{OH}$ group and one of the other three hydrogen bonding groups of Ac-Ala-NHMe are also likely.

Influence of Hydroxy Groups. To elucidate the effect of surface-bound $\mathrm{OH}$ groups on Ac-Ala-NHMe conformational preferences, we performed three-dimensional $\mathrm{ABF}$ calculations using three transition coordinates: $\phi, \psi$, and $d$, the distance in the $x y$ plane between the center of mass of Ac-Ala-NHMe and the center of mass of a single hydroxy group or a pair of hydroxy groups, illustrated in Figure $6 \mathrm{~A}$. Figure $6 \mathrm{~B}, \mathrm{C}$ plots the depth of the free-energy minima associated with $\beta, \beta$-planar, $\alpha$, $\alpha$-planar, and $\alpha_{\mathrm{L}}$ conformations in $(\phi, \psi)$ cross sections of the $w(\phi, \psi, d)$ PMF. At large lateral distances from the $\mathrm{OH}$ groups, the relation between the free energies of the different conformations is similar to that on naked graphene (compare Table 1). Near the hydroxy groups, the overall affinity of AcAla-NHMe for the surface is reduced and all free energies rise, owing to reduced contact between Ac-Ala-NHMe and the $\mathrm{sp}^{2}$ carbon surface. However, the free energies do not all rise in equal amounts. The free energy of the $\beta$ conformation is the least affected by the proximity of the $\mathrm{OH}$. On the other hand, the $\beta$-planar conformation, which is the most favorable on naked graphene, becomes less favorable than the $\beta$ conformation for distances of $d<3.2 \AA$ from the single $\mathrm{OH}$ group and $d<4.4 \AA$ from the pair of $\mathrm{OH}$ groups.

\section{CONCLUSIONS}

In the present work, we have used molecular dynamics simulations and efficient free-energy calculation techniques to reveal, at the atomic scale, details of the interaction between graphenic surfaces and Ac-Ala-NHMe, a simple model of protein backbone structure. We find that the conformation of Ac-Ala-NHMe on these surfaces is predominantly defined by amide $-\pi$ stacking interactions. Correspondingly, the most favorable conformations on the surface are mechanically strained planar conformations characterized by amide $-\pi$ stacking of both amide groups of Ac-Ala-NHMe. The preferred conformations of Ac-Ala-NHMe in water, corresponding to backbone structures similar to those in $\beta$-sheets and $\alpha$-helices, become metastable on the graphene surface, where they are associated with amide $-\pi$ stacking involving one of the two amides, preferably the N-terminal one. Hydrogen bonds to interfacial water molecules or, on hydroxylated graphene, to covalently bound $\mathrm{OH}$ groups play significant roles in stabilizing nonplanar conformations, in addition to amide $-\pi \mathrm{NH}$ face-on interactions. Our free-energy calculations also indicate that the influence of the graphenic surface on the conformation of AcAla-NHMe is particularly short-ranged and appears to require direct contact, while the forces driving adsorption extend farther from the surface (at least to $8 \AA$ ).

Ac-Ala-NHMe is a very basic model, missing many features important for peptide and protein structure, such as the behavior of different side chains or the effects of intramolecular hydrogen bonds, and further studies will be required to understand how these features might alter backbone interaction with graphenic surfaces. However, to our knowledge, the 

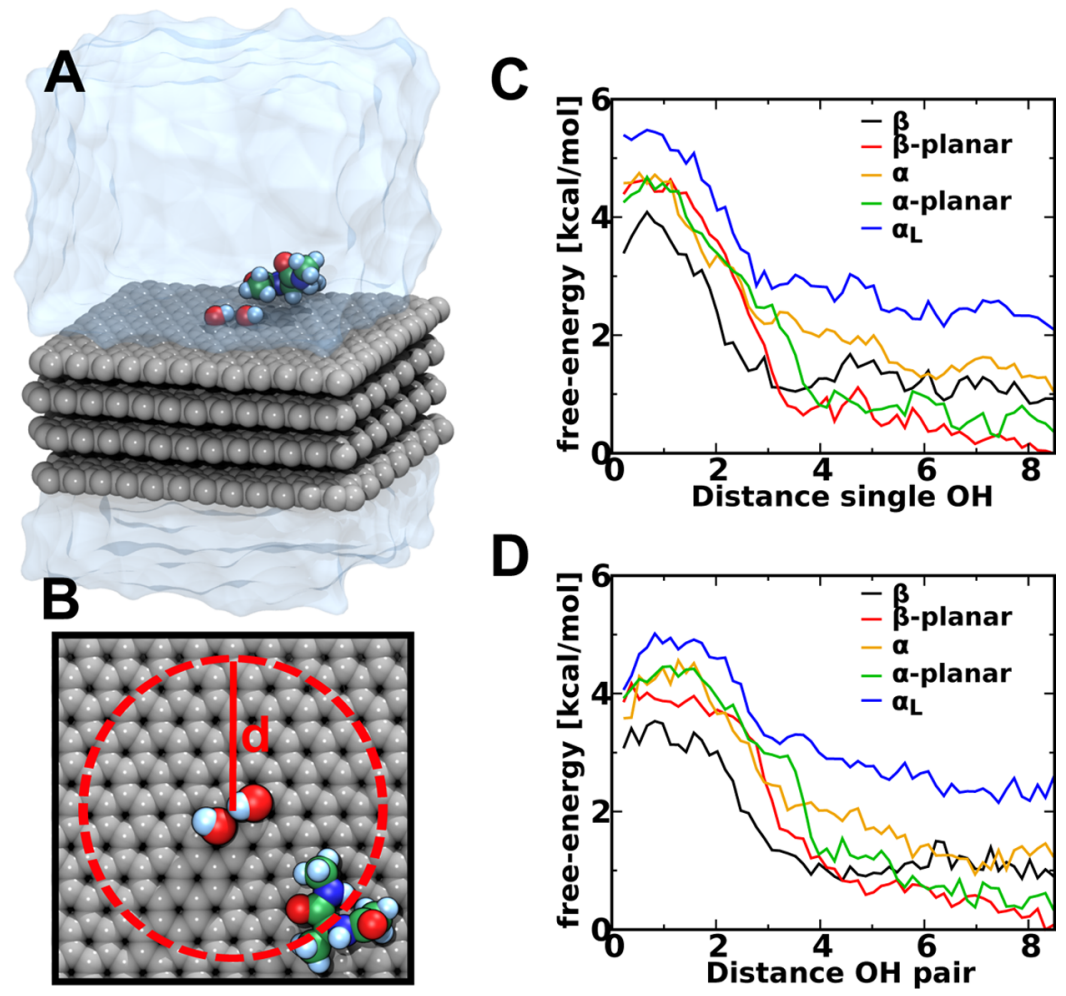

Figure 6. Conformational free-energy landscape as a function of the distance to surface-bound hydroxy groups. (A) Snapshot of the simulation system with a pair of graphene-conjugated hydroxy groups. (B) Diagram of the transition coordinate $d$, the distance projected onto the $x y$ plane between the center of mass of Ac-Ala-NHMe and the center of mass of the hydroxy group(s). (C, D) Free energy of local minima in the Ramachandran landscape as a function of $d$ for a single $\mathrm{OH}$ group (C) or a pair of $\mathrm{OH}$ groups (D).

principal role of amide $-\pi$ stacking in defining the conformational equilibrium of peptides on graphene or carbon nanotubes has not been previously acknowledged and likely has implications for the stability of secondary structure motifs adsorbed to graphene.

\section{ASSOCIATED CONTENT}

\section{S Supporting Information}

The Supporting Information is available free of charge on the ACS Publications website at DOI: 10.1021/acs.jpcb.7b01130.

Details of the estimation of statistical uncertainty in the free energy calculations and additional figures as described in the text (PDF)

Example simulation files (ZIP)

\section{AUTHOR INFORMATION}

\section{Corresponding Author}

*E-mail: jeffcomer@ksu.edu.

\section{ORCID}

Jeffrey Comer: 0000-0003-4437-1260

Notes

The authors declare no competing financial interest.

\section{ACKNOWLEDGMENTS}

This work was partially supported by the Kansas Bioscience Authority funds to the Institute of Computational Comparative Medicine (ICCM) at Kansas State University and to the Nanotechnology Innovation Center of Kansas State University (NICKS). Further financial support derives from the Kansas State University Global Food Systems Innovation Program.
Computing for this project was performed on the Beocat Research Cluster at Kansas State University, which is funded in part by NSF grants CNS-1006860, EPS-1006860, and EPS0919443. This work used the Extreme Science and Engineering Discovery Environment (XSEDE), which is supported by National Science Foundation grant number ACI-1053575.

\section{REFERENCES}

(1) Lundqvist, M.; Stigler, J.; Elia, G.; Lynch, I.; Cedervall, T.; Dawson, K. A. Nanoparticle Size and Surface Properties Determine the Protein Corona with Possible Implications for Biological Impacts. Proc. Natl. Acad. Sci. U. S. A. 2008, 105, 14265-14270.

(2) Aggarwal, P.; Hall, J. B.; McLeland, C. B.; Dobrovolskaia, M. A.; McNeil, S. E. Nanoparticle Interaction with Plasma Proteins as It Relates to Particle Biodistribution, Biocompatibility and Therapeutic Efficacy. Adv. Drug Delivery Rev. 2009, 61, 428-437.

(3) Walczyk, D.; Bombelli, F. B.; Monopoli, M. P.; Lynch, I.; Dawson, K. A. What the Cell "Sees" in Bionanoscience. J. Am. Chem. Soc. 2010, 132, 5761-5768.

(4) Lee, W. C.; Lim, C. H. Y.; Shi, H.; Tang, L. A.; Wang, Y.; Lim, C. T.; Loh, K. P. Origin of Enhanced Stem Cell Growth and Differentiation on Graphene and Graphene Oxide. ACS Nano 2011, 5, 7334-7341.

(5) Monopoli, M. P.; Åberg, C.; Salvati, A.; Dawson, K. A. Biomolecular Coronas Provide the Biological Identity of Nanosized Materials. Nat. Nanotechnol. 2012, 7, 779-786.

(6) Sahneh, F. D.; Scoglio, C. M.; Monteiro-Riviere, N. A.; Riviere, J. E. Predicting the Impact of Biocorona Formation Kinetics on Interspecies Extrapolations of Nanoparticle Biodistribution Modeling. Nanomedicine 2015, 10, 25-33.

(7) Gunawan, C.; Lim, M.; Marquis, C. P.; Amal, R. Nanoparticleprotein Corona Complexes Govern the Biological Fates and Functions of Nanoparticles. J. Mater. Chem. B 2014, 2, 2060-2083. 
(8) Sasidharan, A.; Riviere, J. E.; Monteiro-Riviere, N. Gold and Silver Nanoparticle Interactions with Human Proteins: Impact and Implications in Biocorona Formation. J. Mater. Chem. B 2015, 3, 2075-2082.

(9) Syed, S.; Zubair, A.; Frieri, M. Immune Response to Nanomaterials: Implications for Medicine and Literature Review. Curr. Allergy Asthma Rep. 2013, 13, 50-57.

(10) Mortensen, N. P.; Hurst, G. B.; Wang, W.; Foster, C. M.; Nallathamby, P. D.; Retterer, S. T. Dynamic Development of the Protein Corona on Silica Nanoparticles: Composition and Role in Toxicity. Nanoscale 2013, 5, 6372-6380.

(11) Lee, Y. K.; Choi, E.-J.; Webster, T. J.; Kim, S.-H.; Khang, D. Effect of the Protein Corona on Nanoparticles for Modulating Cytotoxicity and Immunotoxicity. Int. J. Nanomed. 2015, 10, 97.

(12) Dobrovolskaia, M. A.; McNeil, S. E. Immunological Properties of Engineered Nanomaterials. Nat. Nanotechnol. 2007, 2, 469-478.

(13) Salvador-Morales, C.; Flahaut, E.; Sim, E.; Sloan, J.; Green, M. L.; Sim, R. B. Complement Activation and Protein Adsorption by Carbon Nanotubes. Mol. Immunol. 2006, 43, 193-201.

(14) Dobrovolskaia, M. A.; Germolec, D. R.; Weaver, J. L. Evaluation of Nanoparticle Immunotoxicity. Nat. Nanotechnol. 2009, 4, 411-414.

(15) Zolnik, B. S.; González-Fernández, Á.; Sadrieh, N.; Dobrovolskaia, M. A. Minireview: Nanoparticles and the Immune System. Endocrinology 2010, 151, 458-465.

(16) Marucco, A.; Gazzano, E.; Ghigo, D.; Enrico, E.; Fenoglio, I. Fibrinogen Enhances the Inflammatory Response of Alveolar Macrophages to $\mathrm{TiO} 2, \mathrm{SiO} 2$ and Carbon Nanomaterials. Nanotoxicology 2016, 10, 1-9.

(17) Tenzer, S.; Docter, D.; Rosfa, S.; Wlodarski, A.; Kuharev, J.; Rekik, A.; Knauer, S. K.; Bantz, C.; Nawroth, T.; Bier, C.; et al. Nanoparticle Size Is a Critical Physicochemical Determinant of the Human Blood Plasma Corona: a Comprehensive Quantitative Proteomic Analysis. ACS Nano 2011, 5, 7155-7167.

(18) Cedervall, T.; Lynch, I.; Lindman, S.; Berggård, T.; Thulin, E.; Nilsson, H.; Dawson, K. A.; Linse, S. Understanding the Nanoparticleprotein Corona Using Methods to Quantify Exchange Rates and Affinities of Proteins for Nanoparticles. Proc. Natl. Acad. Sci. U. S. A. 2007, 104, 2050-2055.

(19) Dell'Orco, D.; Lundqvist, M.; Oslakovic, C.; Cedervall, T.; Linse, S. Modeling the Time Evolution of the Nanoparticle-protein Corona in a Body Fluid. PLoS One 2010, 5, e10949.

(20) Riviere, J. E. Of Mice, Men and Nanoparticle Biocoronas: Are in Vitro to in Vivo Correlations and Interspecies Extrapolations Realistic? Nanomedicine 2013, 8, 1357-1359.

(21) Sahneh, F. D.; Scoglio, C. M.; Monteiro-Riviere, N. A.; Riviere, J. E. Predicting the Impact of Biocorona Formation Kinetics on Interspecies Extrapolations of Nanoparticle Biodistribution Modeling. Nanomedicine 2015, 10, 25-33.

(22) Lundqvist, M.; Stigler, J.; Cedervall, T.; Flanagan, M. B.; Lynch, I.; Elia, G.; Dawson, K. The Evolution of the Protein Corona Around Nanoparticles: a Test Study. ACS Nano 2011, 5, 7503-7509.

(23) Raesch, S. S.; Tenzer, S.; Storck, W.; Rurainski, A.; Selzer, D.; Ruge, C. A.; Perez-Gil, J.; Schaefer, U. F.; Lehr, C.-M. Proteomic and Lipidomic Analysis of Nanoparticle Corona upon Contact with Lung Surfactant Reveals Differences in Protein, But Not Lipid Composition. ACS Nano 2015, 9, 11872-11885.

(24) Hellstrand, E.; Lynch, I.; Andersson, A.; Drakenberg, T.; Dahlbäck, B.; Dawson, K. A.; Linse, S.; Cedervall, T. Complete Highdensity Lipoproteins in Nanoparticle Corona. FEBS J. 2009, 276, 3372-3381.

(25) Wan, S.; Kelly, P. M.; Mahon, E.; Stockmann, H.; Rudd, P. M.; Caruso, F.; Dawson, K. A.; Yan, Y.; Monopoli, M. P. The "Sweet" Side of the Protein Corona: Effects of Glycosylation on Nanoparticle-cell Interactions. ACS Nano 2015, 9, 2157-2166.

(26) Franz, S.; Rammelt, S.; Scharnweber, D.; Simon, J. C. Immune Responses to Implants-a Review of the Implications for the Design of Immunomodulatory Biomaterials. Biomaterials 2011, 32, 6692-6709.
(27) Park, J. Y.; Davies, J. E. Red Blood Cell and Platelet Interactions with Titanium Implant Surfaces. Clinical Oral Implants Research 2000, $11,530-539$.

(28) Rosengren, A.; Johansson, B. R.; Danielsen, N.; Thomsen, P.; Ericson, L. E. Immunohistochemical Studies on the Distribution of Albumin, Fibrinogen, Fibronectin, IgG and Collagen around PTFE and Titanium Implants. Biomaterials 1996, 17, 1779-1786.

(29) Ulissi, Z. W.; Zhang, J.; Sresht, V.; Blankschtein, D.; Strano, M. S. 2D Equation-of-State Model for Corona Phase Molecular Recognition on Single-Walled Carbon Nanotube and Graphene Surfaces. Langmuir 2015, 31, 628-636.

(30) Comer, J.; Chen, R.; Poblete, H.; Vergara-Jaque, A.; Riviere, J. E. Predicting Adsorption Affinities of Small Molecules on Carbon Nanotubes Using Molecular Dynamics Simulation. ACS Nano 2015, 9, 11761-11774.

(31) Zoungrana, T.; Findenegg, G. H.; Norde, W. Structure, Stability, and Activity of Adsorbed Enzymes. J. Colloid Interface Sci. 1997, 190, 437-448.

(32) Aubin-Tam, M.-E.; Hamad-Schifferli, K. Gold Nanoparticlecytochrome c Complexes: the Effect of Nanoparticle Ligand Charge on Protein Structure. Langmuir 2005, 21, 12080-12084.

(33) Karajanagi, S. S.; Vertegel, A. A.; Kane, R. S.; Dordick, J. S. Structure and Function of Enzymes Adsorbed onto Single-walled Carbon Nanotubes. Langmuir 2004, 20, 11594-11599.

(34) Nakatsu, T.; Ichiyama, S.; Hiratake, J.; Saldanha, A.; Kobashi, N.; Sakata, K.; Kato, H. Structural Basis for the Spectral Difference in Luciferase Bioluminescence. Nature 2006, 440, 372-376.

(35) DeLong, R. K.; Mitchell, J. A.; Morris, R. T.; Comer, J.; Hurst, M. N.; Ghosh, K.; Wanekaya, A.; Mudge, M.; Schaeffer, A.; Washington, L. L.; et al. Enzyme and Cancer Cell Selectivity of Nanoparticles: Inhibition of 3-D Metastatic Phenotype and Experimental Melanoma by Zinc Oxide. J. Biomed. Nanotechnol. 2017, 13, 221-231.

(36) DeLong, R. K.; Hurst, M. N.; Aryal, S.; Inchun, N. K. Unique Boron Carbide Nanoparticle Nanobio Interface: Effects on ProteinRNA Interactions and 3-D Spheroid Metastatic Phenotype. Anticancer Res. 2016, 36, 2097-2104.

(37) Tang, L. Mechanisms of Fibrinogen Domains: Biomaterial Interactions. J. Biomater. Sci., Polym. Ed. 1998, 9, 1257-1266.

(38) Burkett, S. L.; Read, M. J. Adsorption-induced Conformational Changes of $\alpha$-helical Peptides. Langmuir 2001, 17, 5059-5065.

(39) Asuri, P.; Bale, S. S.; Pangule, R. C.; Shah, D. A.; Kane, R. S.; Dordick, J. S. Structure, Function, and Stability of Enzymes Covalently Attached to Single-walled Carbon Nanotubes. Langmuir 2007, 23, 12318-12321.

(40) Jones, L. S.; Peek, L. J.; Power, J.; Markham, A.; Yazzie, B.; Middaugh, C. R. Effects of Adsorption to Aluminum Salt Adjuvants on the Structure and Stability of Model Protein Antigens. J. Biol. Chem. 2005, 280, 13406-13414.

(41) Dong, A.; Jones, L. S.; Kerwin, B. A.; Krishnan, S.; Carpenter, J. F. Secondary Structures of Proteins Adsorbed onto Aluminum Hydroxide: Infrared Spectroscopic Analysis of Proteins from Low Solution Concentrations. Anal. Biochem. 2006, 351, 282-289.

(42) Braun, R.; Sarikaya, M.; Schulten, K. Genetically Engineered Gold-binding Polypeptides: Structure Prediction and Molecular Dynamics. J. Biomater. Sci., Polym. Ed. 2002, 13, 747-757.

(43) Brown, S. Metal-recognition by Repeating Polypeptides. Nat. Biotechnol. 1997, 15, 269-272.

(44) Seker, U. O. S.; Demir, H. V. Material Binding Peptides for Nanotechnology. Molecules 2011, 16, 1426-1451.

(45) Heinz, H.; Farmer, B. L.; Pandey, R. B.; Slocik, J. M.; Patnaik, S. S.; Pachter, R.; Naik, R. R. Nature of Molecular Interactions of Peptides with Gold, Palladium, and Pd-Au Bimetal Surfaces in Aqueous Solution. J. Am. Chem. Soc. 2009, 131, 9704-9714.

(46) Verde, A. V.; Acres, J. M.; Maranas, J. K. Investigating the Specificity of Peptide Adsorption on Gold Using Molecular Dynamics Simulations. Biomacromolecules 2009, 10, 2118-2128.

(47) Palafox-Hernandez, J. P.; Tang, Z.; Hughes, Z. E.; Li, Y.; Swihart, M. T.; Prasad, P. N.; Walsh, T. R.; Knecht, M. R. Comparative 
Study of Materials-Binding Peptide Interactions with Gold and Silver Surfaces and Nanostructures: A Thermodynamic Basis for Biological Selectivity of Inorganic Materials. Chem. Mater. 2014, 26, 4960-4969.

(48) Hughes, Z. E.; Walsh, T. R. What Makes a Good Graphenebinding Peptide? Adsorption of Amino Acids and Peptides at Aqueous Graphene Interfaces. J. Mater. Chem. B 2015, 3, 3211-3221.

(49) Brandt, E. G.; Lyubartsev, A. P. Molecular Dynamics Simulations of Adsorption of Amino Acid Side Chain Analogues and a Titanium Binding Peptide on the TiO2 (100) Surface. J. Phys. Chem. C 2015, 119, 18126-18139.

(50) Zeng, Q.; Jiang, X.; Yu, A.; Lu, G. M. Growth Mechanisms of Silver Nanoparticles: a Molecular Dynamics Study. Nanotechnology 2007, 18, 035708.

(51) Alarcon, E. I.; Udekwu, K.; Skog, M.; Pacioni, N. L.; Stamplecoskie, K. G.; González-Béjar, M.; Polisetti, N.; Wickham, A.; Richter-Dahlfors, A.; Griffith, M.; et al. The Biocompatibility and Antibacterial Properties of Collagen-stabilized, Photochemically Prepared Silver Nanoparticles. Biomaterials 2012, 33, 4947-4956.

(52) Li, Y.; Tang, Z.; Prasad, P. N.; Knecht, M. R.; Swihart, M. T. Peptide-mediated Synthesis of Gold Nanoparticles: Effects of Peptide Sequence and Nature of Binding on Physicochemical Properties. Nanoscale 2014, 6, 3165-3172.

(53) Alarcon, E. I.; Bueno-Alejo, C. J.; Noel, C. W.; Stamplecoskie, K. G.; Pacioni, N. L.; Poblete, H.; Scaiano, J. Human Serum Albumin as Protecting Agent of Silver Nanoparticles: Role of the Protein Conformation and Amine Groups in the Nanoparticle Stabilization. J. Nanopart. Res. 2013, 15, 1-14.

(54) Poblete, H.; Agarwal, A.; Thomas, S. S.; Bohne, C.; Phospase, J.; Comer, J.; Alarcon, E. I. Peptide-Silver Nanoparticle Interaction: Deciphering the Role of Cysteine and Lysine on Nanoparticle Stability. Langmuir 2016, 32, 265-273.

(55) Derr, L.; Hildebrand, N.; Köppen, S.; Kunze, S.; Treccani, L.; Dringen, R.; Rezwan, K.; Ciacchi, L. C. Physisorption of $\alpha$ chymotrypsin on $\mathrm{SiO} 2$ and $\mathrm{TiO} 2$ : A Comparative Study via Experiments and Molecular Dynamics Simulations. Biointerphases 2016, 11, 011007.

(56) Brancolini, G.; Kokh, D. B.; Calzolai, L.; Wade, R. C.; Corni, S. Docking of Ubiquitin to Gold Nanoparticles. ACS Nano 2012, 6, 9863-9878.

(57) Carr, R.; Comer, J.; Ginsberg, M. D.; Aksimentiev, A. Modeling Pressure-Driven Transport of Proteins through a Nanochannel. IEEE Trans. Nanotechnol. 2011, 10, 75-82.

(58) Kubelka, J.; Hofrichter, J.; Eaton, W. A. The Protein Folding 'speed Limit. Curr. Opin. Struct. Biol. 2004, 14, 76-88.

(59) Freddolino, P.; Harrison, C.; Liu, Y.; Schulten, K. Challenges in Protein-folding Simulations. Nat. Phys. 2010, 6, 751-758.

(60) Lindorff-Larsen, K.; Piana, S.; Dror, R. O.; Shaw, D. E. How Fast-folding Proteins Fold. Science 2011, 334, 517-520.

(61) Huang, X.; Bowman, G. R.; Pande, V. S. Convergence of Folding Free Energy Landscapes via Application of Enhanced Sampling Methods in a Distributed Computing Environment. J. Chem. Phys. 2008, 128, 205106.

(62) Pietrucci, F.; Laio, A. A Collective Variable for the Efficient Exploration of Protein Beta-sheet Structures: Application to SH3 and GB1. J. Chem. Theory Comput. 2009, 5, 2197-2201.

(63) Hazel, A.; Chipot, C.; Gumbart, J. C. Thermodynamics of Decaalanine Folding in Water. J. Chem. Theory Comput. 2014, 10, 28362844.

(64) Miao, Y.; Feher, V. A.; McCammon, J. A. Gaussian Accelerated Molecular Dynamics: Unconstrained Enhanced Sampling and Free Energy Calculation. J. Chem. Theory Comput. 2015, 11, 3584-3595.

(65) Meißner, R. H.; Schneider, J.; Schiffels, P.; Colombi Ciacchi, L. Computational Prediction of Circular Dichroism Spectra and Quantification of Helicity Loss upon Peptide Adsorption on Silica. Langmuir 2014, 30, 3487-3494.

(66) Loh, K. P.; Bao, Q.; Eda, G.; Chhowalla, M. Graphene Oxide as a Chemically Tunable Platform for Optical Applications. Nat. Chem. 2010, 2, 1015-1024.
(67) Liu, J.; Cui, L.; Losic, D. Graphene and Graphene Oxide as New Nanocarriers for Drug Delivery Applications. Acta Biomater. 2013, 9, 9243-9257.

(68) Chung, C.; Kim, Y.-K.; Shin, D.; Ryoo, S.-R.; Hong, B. H.; Min, D.-H. Biomedical Applications of Graphene and Graphene Oxide. Acc. Chem. Res. 2013, 46, 2211-2224.

(69) Lee, J.; Kim, J.; Kim, S.; Min, D.-H. Biosensors Based on Graphene Oxide and Its Biomedical Application. Adv. Drug Delivery Rev. 2016, 105, 275.

(70) Segal, M. Selling Graphene by the Ton. Nat. Nanotechnol. 2009, 4, 612-614.

(71) Zurutuza, A.; Marinelli, C. Challenges and Opportunities in Graphene Commercialization. Nat. Nanotechnol. 2014, 9, 730-734.

(72) De Volder, M. F.; Tawfick, S. H.; Baughman, R. H.; Hart, A. J. Carbon Nanotubes: Present and Future Commercial Applications. Science 2013, 339, 535-539.

(73) Hu, W.; Peng, C.; Lv, M.; Li, X.; Zhang, Y.; Chen, N.; Fan, C.; Huang, Q. Protein Corona-mediated Mitigation of Cytotoxicity of Graphene Oxide. ACS Nano 2011, 5, 3693-3700.

(74) Ge, C.; Du, J.; Zhao, L.; Wang, L.; Liu, Y.; Li, D.; Yang, Y.; Zhou, R.; Zhao, Y.; Chai, Z.; et al. Binding of Blood Proteins to Carbon Nanotubes Reduces Cytotoxicity. Proc. Natl. Acad. Sci. U. S. A. 2011, 108, 16968-16973.

(75) Li, Y.; Yuan, H.; von dem Bussche, A.; Creighton, M.; Hurt, R. H.; Kane, A. B.; Gao, H. Graphene Microsheets Enter Cells through Spontaneous Membrane Penetration at Edge Asperities and Corner Sites. Proc. Natl. Acad. Sci. U. S. A. 2013, 110, 12295-12300.

(76) Vanommeslaeghe, K.; Hatcher, E.; Acharya, C.; Kundu, S.; Zhong, S.; Shim, J.; Darian, E.; Guvench, O.; Lopes, P.; Vorobyov, I.; et al. CHARMM General Force Field: A Force Field for Drug-like Molecules Compatible with the CHARMM All-atom Additive Biological Force Fields. J. Comput. Chem. 2010, 31, 671-690.

(77) Hughes, Z. E.; Tomásio, S. M.; Walsh, T. R. Efficient Simulations of the Aqueous Bio-interface of Graphitic Nanostructures with a Polarisable Model. Nanoscale 2014, 6, 5438-5448.

(78) Rossky, P. J.; Karplus, M. Solvation. A Molecular Dynamics Study of a Dipeptide in Water. J. Am. Chem. Soc. 1979, 101, 19131937.

(79) Tobias, D. J.; Brooks, C. L., III Conformational Equilibrium in the Alanine Dipeptide in the Gas Phase and Aqueous Solution: A Comparison of Theoretical Results. J. Phys. Chem. 1992, 96, 38643870.

(80) Chipot, C.; Pohorille, A. Conformational Equilibria of Terminally Blocked Single Amino Acids at the Water-hexane Interface. A Molecular Dynamics Study. J. Phys. Chem. B 1998, 102, 281-290.

(81) Smith, P. E. The Alanine Dipeptide Free Energy Surface in Solution. J. Chem. Phys. 1999, 111, 5568-5579.

(82) Parchanšký, V.; Kapitán, J.; Kaminský, J.; Šebestík, J.; Boư̌, P. Ramachandran Plot for Alanine Dipeptide as Determined from Raman Optical Activity. J. Phys. Chem. Lett. 2013, 4, 2763-2768.

(83) Feig, M. Is Alanine Dipeptide a Good Model for Representing the Torsional Preferences of Protein Backbones? J. Chem. Theory Comput. 2008, 4, 1555-1564.

(84) Ramachandran, G.; Ramakrishnan, C.; Sasisekharan, V. Stereochemistry of Polypeptide-chain Configurations. J. Mol. Biol. 1963, 7, 95-99.

(85) MacKerell, A. D., Jr.; Bashford, D.; Bellott, M.; Dunbrack, R. L., Jr.; Evanseck, J. D.; Field, M. J.; Fischer, S.; Gao, J.; Guo, H.; Ha, S.; et al. All-atom Empirical Potential for Molecular Modeling and Dynamics Studies of Proteins. J. Phys. Chem. B 1998, 102, 3586-3616. (86) Best, R. B.; Zhu, X.; Shim, J.; Lopes, P. E.; Mittal, J.; Feig, M.; MacKerell, A. D., Jr Optimization of the Additive CHARMM All-atom Protein Force Field Targeting Improved Sampling of the Backbone $\phi$, $\psi$ and Side-chain $\chi 1$ and $\chi 2$ Dihedral Angles. J. Chem. Theory Comput. 2012, 8, 3257-3273.

(87) MacKerell, A., Jr; Feig, M.; Brooks, C., III Improved Treatment of the Protein Backbone in Empirical Force Fields. J. Am. Chem. Soc. 2004, 126, 698-699. 
(88) Smith, T. J. MolView: a Program for Analyzing and Displaying Atomic Structures on the Macintosh Personal Computer. J. Mol. Graphics 1995, 13, 122-125.

(89) Vanommeslaeghe, K.; MacKerell, A. D., Jr Automation of the CHARMM General Force Field (CGenFF) I: Bond Perception and Atom Typing. J. Chem. Inf. Model. 2012, 52, 3144-3154.

(90) Vanommeslaeghe, K.; Raman, E. P.; MacKerell, A. D., Jr Automation of the CHARMM General Force Field (CGenFF) II: Assignment of Bonded Parameters and Partial Atomic Charges. J. Chem. Inf. Model. 2012, 52, 3155-3168.

(91) Humphrey, W.; Dalke, A.; Schulten, K. VMD - Visual Molecular Dynamics. J. Mol. Graphics 1996, 14, 33-38.

(92) Kohlmeyer, A. TopoTools, release 1.7; 2016. DOI: 10.5281/ zenodo.50249.

(93) Phillips, J. C.; Braun, R.; Wang, W.; Gumbart, J.; Tajkhorshid, E.; Villa, E.; Chipot, C.; Skeel, R. D.; Kale, L.; Schulten, K. Scalable Molecular Dynamics with NAMD. J. Comput. Chem. 2005, 26, 17811802.

(94) Feller, S. E.; Zhang, Y. H.; Pastor, R. W.; Brooks, B. R. Constant Pressure Molecular Dynamics Simulations - The Langevin Piston Method. J. Chem. Phys. 1995, 103, 4613-4621.

(95) Darden, T. A.; York, D. M.; Pedersen, L. G. Particle Mesh Ewald: An $N \log N$ Method for Ewald Sums in Large Systems. J. Chem. Phys. 1993, 98, 10089-10092.

(96) Miyamoto, S.; Kollman, P. A. SETTLE: an Analytical Version of the SHAKE and RATTLE Algorithm for Rigid Water Models. J. Comput. Chem. 1992, 13, 952-962.

(97) Andersen, H. C. RATTLE: A "Velocity" Version of the SHAKE Algorithm for Molecular Dynamics Calculations. J. Comput. Phys. 1983, 52, 24-34.

(98) Hopkins, C. W.; Le Grand, S.; Walker, R. C.; Roitberg, A. E. Long-time-step Molecular Dynamics through Hydrogen Mass Repartitioning. J. Chem. Theory Comput. 2015, 11, 1864-1874.

(99) Towns, J.; Cockerill, T.; Dahan, M.; Foster, I.; Gaither, K.; Grimshaw, A.; Hazlewood, V.; Lathrop, S.; Lifka, D.; Peterson, G. D.; et al. XSEDE: Accelerating Scientific Discovery. Comput. Sci. Eng. 2014, 16, 62-74.

(100) Comer, J.; Gumbart, J. C.; Hénin, J.; Lelièvre, T.; Pohorille, A.; Chipot, C. The Adaptive Biasing Force Method: Everything You Always Wanted to Know But Were Afraid to Ask. J. Phys. Chem. B 2015, 119, 1129-1151.

(101) Darve, E.; Rodríguez-Gómez, D.; Pohorille, A. Adaptive Biasing Force Method for Scalar and Vector Free Energy Calculations. J. Chem. Phys. 2008, 128, 144120.

(102) Darve, E.; Pohorille, A. Calculating Free Energies Using Average Force. J. Chem. Phys. 2001, 115, 9169-9183.

(103) Hénin, J.; Fiorin, G.; Chipot, C.; Klein, M. Exploring Multidimensional Free Energy Landscapes Using Time-dependent Biases on Collective Variables. J. Chem. Theory Comput. 2010, 6, 3547.

(104) Fiorin, G.; Klein, M. L.; Hénin, J. Using Collective Variables to Drive Molecular Dynamics Simulations. Mol. Phys. 2013, 111, 33453362.

(105) Ensing, B.; De Vivo, M.; Liu, Z.; Moore, P.; Klein, M. L. Metadynamics as a Tool for Exploring Free Energy Landscapes of Chemical Reactions. Acc. Chem. Res. 2006, 39, 73-81.

(106) Esque, J.; Cecchini, M. Accurate Calculation of Conformational Free Energy Differences in Explicit Water: The ConfinementSolvation Free Energy Approach. J. Phys. Chem. B 2015, 119, 51945207.

(107) Imai, Y. N.; Inoue, Y.; Nakanishi, I.; Kitaura, K. Amide- $\pi$ Interactions between Formamide and Benzene. J. Comput. Chem. 2009, 30, 2267-2276.

(108) Hansen, N.; Van Gunsteren, W. F. Practical Aspects of Freeenergy Calculations: a Review. J. Chem. Theory Comput. 2014, 10, 2632-2647.

(109) Jo, S.; Chipot, C.; Roux, B. Efficient Determination of Relative Entropy Using Combined Temperature and Hamiltonian Replica- exchange Molecular Dynamics. J. Chem. Theory Comput. 2015, 11, 2234-2244.

(110) Cai, W.; Piner, R. D.; Stadermann, F. J.; Park, S.; Shaibat, M. A.; Ishii, Y.; Yang, D.; Velamakanni, A.; An, S. J.; Stoller, M.; et al. Synthesis and Solid-state NMR Structural Characterization of 13Clabeled Graphite Oxide. Science 2008, 321, 1815-1817.

(111) Bagri, A.; Mattevi, C.; Acik, M.; Chabal, Y. J.; Chhowalla, M.; Shenoy, V. B. Structural Evolution during the Reduction of Chemically Derived Graphene Oxide. Nat. Chem. 2010, 2, 581-587.

(112) Hunt, A.; Dikin, D. A.; Kurmaev, E. Z.; Boyko, T. D.; Bazylewski, P.; Chang, G. S.; Moewes, A. Epoxide Speciation and Functional Group Distribution in Graphene Oxide Paper-Like Materials. Adv. Funct. Mater. 2012, 22, 3950-3957. 